\title{
Die Schultersteife
}

\author{
F. Dehlinger, B. Hollinger, T. Ambacher
}

Der Begriff Schultersteife beschreibt nicht nur einen Zustand, sondern auch eine Erkrankung des Schultergelenks mit Einschränkung der Beweglichkeit in allen Ebenen. Verantwortlich dafür ist eine Fibrosierung der Gelenkkapsel.

Bei der Schultersteife wird eine primäre von einer sekundären Form unterschieden. Bei der primären oder idiopathischen Schultersteife kommt es aus unbekannten Ursachen zu einem typischen Verlauf in 3 Phasen mit unterschiedlich stark ausgeprägter Steifigkeit und Schmerzsymptomatik. Eine vollständige Ausheilung ist nach einer Krankheitsdauer von einigen Monaten bis wenigen Jahren möglich. Die sekundäre Steife entwickelt sich nach Verletzungen, Operationen oder im Rahmen anderer Schulter- oder systemischer Erkrankungen. Die Phasen sind oft nicht abgrenzbar, und es verbleiben öfter Restbeschwerden.

Klinisch zeigt sich eine eingeschränkte glenohumerale Beweglichkeit, zunächst der Außenrotation, später auch der Flexion, Abduktion und Innenrotation bei gestörtem skapu- lothorakalem Rhythmus. Zu Beginn bestehen ausgeprägte bewegungsabhängige und Ruheschmerzen. Röntgendiagnostik und Ultraschall sind unauffällig, im MRT können sich Hinweise auf die Erkrankung ergeben.

Die Therapie erfolgt stadienadaptiert, im Anfangsstadium steht die Schmerztherapie im Vordergrund, Interventionen sind kontraindiziert. In der 1. und 2. Phase kann eine Behandlung mit oralen Steroiden mit guten Erfolgsaussichten erfolgen. Bei rückläufigen Schmerzen im weiteren Verlauf werden zunehmend Physiotherapie und eigenständige Dehnübungen eingesetzt. Bei anhaltenden und inakzeptablen Beschwerden in der späten 2. und 3. Phase sowie bei der sekundären Steife ohne Besserung auf konservative Maßnahmen besteht die Indikation zur arthroskopischen Arthrolyse, wobei eine Kapsulotomie durchgeführt wird. Für eine offene Arthrolyse besteht dagegen keine Indikation mehr. Mit anschließender intensiver Physiotherapie lassen sich auf diese Weise gute Ergebnisse erzielen.

\section{Historischer Rückblick}

Ende des 19. Jahrhunderts wurde von Duplay der Begriff der „Periarthritis humeroscapularis“ geprägt, unter dem eine Vielzahl von Schulterpathologien inklusive der steifen Schulter zusammengefasst wurden. Von Codman stammte dann 1934 der Begriff der "frozen shoulder“ als Bezeichnung für eine eingeschränkte Beweglichkeit des Gelenks mit noch unspezifischer Definition. Auch wenn zunächst Probleme im subakromialen Gleitraum als Ursache der Erkrankung vermutet wurden, zeigte sich bald, dass die zugrunde liegende Pathologie nicht dort, sondern im Bereich der Kapsel liegt. Gut 10 Jahre später folgte von Neviaser der Ausdruck „adhäsive Kapsulitis“, der die von der Kapsel ausgehende und entzündlich bedingte Komponente der Erkrankung betont.

Trotz der üblichen Verwendung des Begriffs einer adhäsiven Kapsulitis oder auch eines adhäsiven Subakromialsyndroms sind eigentliche Adhäsionen, z. B. zwischen Knorpeloberfläche und Synovia, nicht vorhanden.

\section{Abkürzungen}

$\begin{array}{ll}\text { ACG } & \text { Akromioklavikulargelenk } \\ \text { CPM } & \text { continuous passive motion } \\ \text { CRPS } & \text { chronic regional pain syndrome } \\ \text { GIRD } & \text { glenohumerales Innenrotationsdefizit } \\ \text { HGF } & \text { hepatocyte growth factor } \\ \text { HLA } & \text { human leucocyte antigen } \\ \text { IgA } & \text { Immunglobulin A } \\ \text { IGF2 } & \text { insulin-like growth factor 2 } \\ \text { IGHL } & \text { inferiores glenohumerales Ligament } \\ \text { ISP } & \text { Infraspinatus } \\ \text { MGHL } & \text { mittleres glenohumerales Ligament } \\ \text { NSAID } & \text { nichtsteroidale Antiphlogistika } \\ \text { PDGF } & \text { platelet-derived growth factor } \\ \text { SGHL } & \text { superiores glenohumerales Ligament } \\ \text { SSC } & \text { Subscapularis } \\ \text { SSP } & \text { Supraspinatus } \\ \text { TENS } & \text { transkutane elektrische Nervenstimulation } \\ \text { TGF- } \beta & \text { transforming growth factor } \beta\end{array}$


Des Weiteren bestehen auch keine floriden entzündlichen Infiltrate und keine Exsudation.

- Es handelt sich bei der Schultersteife vielmehr um eine chronisch entzündliche Veränderung mit Verdickung und Kontraktur bzw. zuletzt Fibrosierung des Kapselgewebes mit konsekutiv vermindertem Gelenkvolumen und entsprechend verringerter Bewegungsfähigkeit der beiden Gelenkpartner.

\section{Klassifikation}

Es ist sinnvoll, eine primäre von einer sekundären Form der Schultersteife zu unterscheiden. Bei der primären oder idiopathischen Schultersteife kommt es zu einer Fibrosierung der Weichteile des Glenohumeralgelenks aus unbekannter Ursache mit häufig typischem Verlauf. Die sekundäre Form hingegen folgt einem spezifischen Auslöser, meistens einem Makrotrauma, aber auch rezidivierende Mikrotraumata können ursächlich sein. Operationen bzw. postoperative Veränderungen und andere Schultergelenkerkrankungen wie Rotatorenmanschettendefekte oder die Tendinosis calcarea können ebenfalls eine sekundäre Steifigkeit des Gelenks verursachen. Im englischen Sprachraum wird diese Unterscheidung verdeutlicht, indem die primäre Form als „frozen shoulder“ und die sekundäre als „shoulder stiffness“ bezeichnet wird.

Beide Arten der Schultersteife haben Gemeinsamkeiten, aber auch Unterschiede, die im Folgenden näher erläutert werden.

\section{Primäre Schultersteife}

Ursache und genaue Mechanismen der adhäsiven Kapsulitis des Schultergelenks sind immer noch weitgehend unbekannt. Es wird heute davon ausgegangen, dass die Erkrankung im Bereich des Rotatorenintervalls und des Lig. coracohumerale beginnt und sich dann über die gesamte ventrale Kapsel bis nach kaudal und dorsal ausbreitet. Pathophysiologisch wurde zunächst vermehrt Kollagen im Kapselgewebe und ein erhöhter Proteoglykangehalt festgestellt, eine akute Entzündung und Beteiligung des synovialen Gewebes liegt nicht immer bzw. nur zu Beginn der Erkrankung vor. Lymphzellinfiltrate und verminderte IgA-Serumspiegel deuteten auf Autoimmunprozesse hin, immunologische Tests für die Diagnose oder Therapie der frozen shoulder existieren jedoch nicht.

\begin{tabular}{l} 
Übersicht \\
\hline „Schultersteife“ \\
\hline - primäre/idiopathische Schultersteife \\
(adhäsive Kapsulitis, frozen shoulder) \\
- sekundäre Schultersteife (shoulder stiffness) \\
- posttraumatisch \\
- postoperativ \\
- bei primär anderer Schultererkrankung \\
(Subakromialsyndrom, Rotatorenmanschettendefekt, \\
Tendinosis calcarea etc.)
\end{tabular}

Eine HLA-B27-Assoziation wurde teilweise nachgewiesen, in anderen Studien aber auch bestritten. Histologisch zeigen sich eine aktive Fibroblastenproliferation, Kollagenknoten und eine Myofibroblastentransformation, sodass die Theorie zur Erkrankung, die heute von den meisten Autoren gestützt wird, eine Fibrose der Gelenkkapsel ist.

An den frühen entzündlichen Stadien sind Zytokine wie TGF- $\beta$ („transforming growth factor“), PDGF (,plateletderived growth factor“) und HGF („hepatocyte growth factor") beteiligt. Wie beim Morbus Dupuytren finden sich erhöhte $\beta$-Catenin- und IGF2-Spiegel in Gewebeproben bei der primären Schultersteife.

Es existieren diverse Definitionen der Schultersteife. Da Unklarheiten bestehen, ob die Beweglichkeit des Armes gegenüber dem Körper oder isoliert des Humerus gegenüber der (fixierten) Skapula gemessen und welche bzw. wie viele Bewegungsebenen zur Diagnose herangezogen werden sollten, gibt es keine allgemeingültigen, das Ausmaß der Bewegungseinschränkung betreffenden Kriterien.

- Bei einer Limitierung der Flexion auf $90-110^{\circ}$ kann vom Vollbild einer Schultersteife gesprochen werden.

- Für mittelschwere Befunde wurde eine Reduktion der Innenrotation von $10-15^{\circ}$, der Abduktion von $45^{\circ}$ sowie der Außenrotation von $60-70^{\circ}$ beschrieben.

- Nicht allgemein definiert oder in der Literatur beschrieben, aber im klinischen Sprachgebrauch doch verwendbar ist der Begriff der Teilschultersteife (oder Schulterteilsteife), der mit einer Einschränkung der Flexion/Elevation auf $120-150^{\circ}$ verbunden ist. Im Gegensatz zum Begriff der Blockierung sind alle Bewegungsebenen betroffen. 


\section{Checkliste}

\section{Diagnostische Kriterien der primären/ idiopathischen Schultersteife nach Kessel (1982)}

anamnestisch eingeschränkte Schulterbeweg-
lichkeit ohne relevantes Trauma oder Operation
in der Vorgeschichte
klinisch Steifigkeit in allen Ebenen (ohne Verlust
von Kraft, Stabilität oder glatten Gelenkober-
flächen)
unauffällige Röntgenaufnahmen mit normalem
Gelenkspalt und ohne fokale periartikuläre
Abnormitäten (Osteopenie möglich)

Von Kessel (1982) wurden notwendige und ausreichende diagnostische Kriterien für eine idiopathische Schultersteife beschrieben (s. Checkliste).

\section{Epidemiologie}

Die Inzidenz liegt bei ca. $2 \%$ der Bevölkerung, die Erkrankung tritt gehäuft bei Frauen zwischen dem 40. und dem 60. Lebensjahr auf, hier steigt die Inzidenz auf bis zu $5 \%$. Das Durchschnittsalter bei Männern und Frauen liegt bei ca. 50 Jahren. Die Häufung bei Frauen um die Menopause legt einen hormonellen Zusammenhang nahe, dieser konnte aber bisher nicht eindeutig nachgewiesen werden.

Ein beidseitiges Auftreten wird - in der Regel metachron - in 20-30\% der Fälle beobachtet, hingegen werden Rezidive nach ausgeheilter Kapsulitis nicht beschrieben.

Eine Reihe von internistischen Erkrankungen wird gehäuft bei weiblichen und männlichen Patienten mit adhäsiver Kapsulitis beobachtet (s. Infobox). Insbesondere besteht eine hohe Koinzidenz mit Diabetes mellitus mit bis zu 30\% (insulinabhängig > nicht insulinabhängig).

Eine primäre Schultersteife ist die häufigste diabetische Begleiterkrankung des Bewegungsapparates an der oberen Extremität und kann die Erstmanifestation des Diabetes sein. Umgekehrt ist eine gute Einstellung des Diabetes mellitus ein wichtiger Baustein der Behandlung einer diabetischen Schultersteife.

\section{Übersicht}

\section{Mit Kapsulitis assoziierte Begleiterkrankungen}

- Diabetes mellitus

- Schilddrüsenfunktionsstörungen (Über- und Unterfunktion)

- Fettstoffwechselstörungen

- Apoplex

- Morbus Dupuytren

Das gehäufte gleichzeitige Auftreten eines Morbus Dupuytren mit auch ähnlichen histologischen Veränderungen einer vermehrten Fibrosierung des Bindegewebes gibt Hinweise auf eine systemische Komponente der Krankheit.

Auch wenn in wenigen Studien psychiatrische Erkrankungen wie Depressionen und Angststörungen gehäuft zu finden waren, sind bei den Patienten mit primärer Schultersteife meist keine Unterschiede zur Normalbevölkerung festzustellen. Ob mögliche psychische Veränderungen Ursache oder Folge der Schmerzen sind, ist oft nicht zu klären.

\section{Stadien}

Die primäre Schultersteife verläuft klassischerweise in 3 Stadien, die erstmalig von Reeves (1975) beschrieben wurden (Abb. 1).

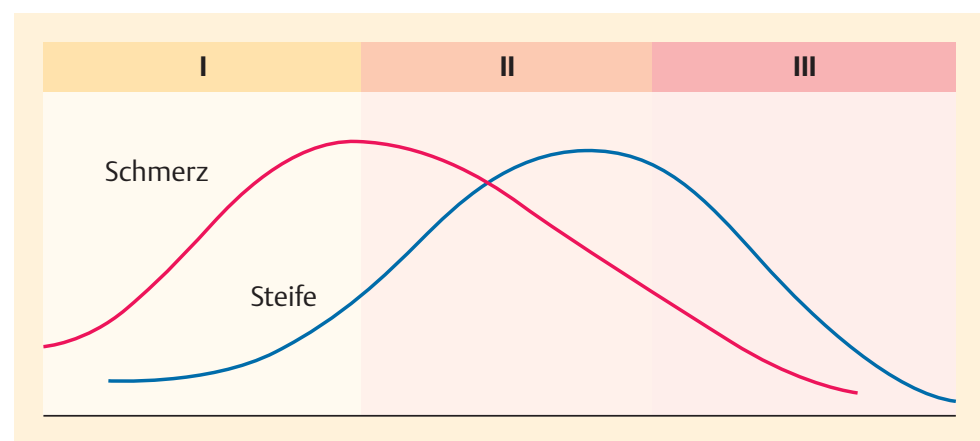

Abb. 1 - Schematisierter, zeitlich versetzter Verlauf von Schmerz und Bewegungseinschränkung bei den 3 Phasen der frozen shoulder. 
- Die 1. Phase ist durch zunehmende Schulterschmerzen gekennzeichnet, die oft plötzlich innerhalb von Tagen bis Wochen beginnen. Im Gegensatz zur sekundären Steife finden sich kein Makrotrauma und keine Operation als Auslöser. Aus einem Kausalitätsbedürfnis heraus werden manchmal Bagatelltraumata oder besondere Belastungen von den Patienten angegeben. Das Ausmaß der Bewegungseinschränkung des Gelenks nimmt langsam zu. Die Dauer der ersten Phase beträgt 2-10 Monate.

- In der 2. Phase erreicht die Steifigkeit ihren Höhepunkt, die Schmerzen lassen langsam nach. Die Behinderung durch die fehlende Beweglichkeit steht im Vordergrund, Ruheschmerzen sind nicht mehr vorhanden, aber noch bewegungsabhängige Schmerzen. Die 2. Phase dauert 3-12 Monate.

- Die letzte Phase ist durch eine langsame Besserung der Beweglichkeit und weiter rückläufige Schmerzen gekennzeichnet, sie kann 5-24 Monate dauern, selten auch länger.

Im Englischen werden diese Stadien der primären Schultersteife plastisch mit „freezing“, „frozen“ und „thawing phase“ beschrieben.

Eine vollständige Wiederherstellung - Restitutio ad integrum - ist bei der adhäsiven Kapsulitis häufig möglich. Verbesserte, aber noch vorhandene Bewegungseinschränkungen und Schmerzen mit unterschiedlicher Ausprägung können jedoch auch lange oder dauerhaft verbleiben. In Studien wurde ein Anteil von 20-30\% Restbeschwerden festgestellt, dieser Anteil ist bei der sekundären Schultersteife höher.

\section{Sekundäre Schultersteife}

\section{Posttraumatisch}

Die sekundäre Form der Schultersteife entspricht mehr einer Kombination unterschiedlicher Ursachen. Für diese Form der Schultersteife liegen deutlich weniger epidemiologische Daten vor.

Die Bewegungseinschränkung nach einem Trauma oder einer Operation kann Adhäsionen in den extraartikulären humeroskapulären Kontaktflächen, Kontrakturen der muskulotendinösen Einheiten oder eine Fibrosierung der Gelenkkapsel anzeigen. Ist bei der primären Schultersteife der subakromiale Gleitraum häufig unauffällig bzw. am Krankheitsbild nicht beteiligt, finden sich bei den sekundären Formen hier oft Adhäsionen.
Typische auslösende Verletzungen sind

- proximale Humerusfrakturen,

- schwere Weichteilverletzungen und

- Rotatorenmanschettenrupturen, oft verbunden mit einer langen Ruhigstellungsdauer.

Nach schultergelenknahen Humerusfrakturen führt die bindegewebige Umwandlung des Hämatoms zu einer Bewegungseinschränkung. Hier besteht ein Zusammenhang zum Alter des Patienten: Das Risiko einer posttraumatischen Schultersteife steigt mit zunehmendem Lebensalter deutlich an.

Postoperativ entstehen Vernarbungen im Bereich der operativen Zugangswege, z.B. an der SSC-Sehne nach ventraler Arthrotomie mit SSC-Tenotomie. Teils ist jedoch vor allem nach offenen Eingriffen auch der komplette subdeltoidale Gleitraum praktisch von Narbengewebe ausgemauert.

\section{Außerdem kommt es durch eine Ruhigstellung des} Gelenks (schmerzbedingt nach Trauma oder Operation, iatrogen verordnet bei konservativer Frakturtherapie oder postoperativ, aber auch z.B. im Rahmen einer Langzeitbeatmung) zu einer Kapselschrumpfung, die sich nur langsam oder gar nicht mehr zurückbildet. Dies scheint sich beim Schultergelenk mit seinem großen Bewegungsumfang und seinen weiten Recessus articularis relativ schnell und deutlich zu manifestieren.

- Der Verlauf der sekundären Schultersteife orientiert sich nicht an den 3 Phasen der frozen shoulder. Die Dauer der Erkrankung ist weniger gut vorherzusagen, und es verbleiben häufiger Einschränkungen und Restbeschwerden.

Auch nach Luxationen kann sich im Verlauf eine posttraumatische Schultersteife entwickeln. Stellt sich ein jüngerer Patient 4-6 Wochen nach einer Luxation mit den typischen Folgen im MRT (Bankart-Läsion, HillSachs-Delle, keine Rotatorenmanschettenruptur) und mit anhaltenden oder zunehmenden Schmerzen vor, und es findet sich passiv eine eingeschränkte Beweglichkeit, sollte auf jeden Fall von einer Stabilisierung Abstand genommen werden. Das Rezidivluxationsrisiko ist bei diesen Patienten als sehr gering einzustufen, die krankengymnastische Behandlung zur Wiederherstellung eines normalen Bewegungsumfangs steht hier klar im Vordergrund. Bei fehlenden Fortschritten ist bei diesen Patienten dann entsprechend eine arthroskopische Arthrolyse und keine Stabilisierung indiziert. 
Manchmal lassen sich aber auch makroskopisch bzw. im MRT nach einem direkten oder indirekten Trauma des Schultergelenks keine Verletzungsfolgen feststellen, und trotzdem kann es nach zunächst nur geringen Schmerzen Tage bis Wochen nach dem Trauma zu zunehmenden Schmerzen und dann auch Bewegungseinschränkungen wie bei der klassischen frozen shoulder kommen. Warum und wie dieser Reiz als Auslöser funktioniert,

ist nicht bekannt, die Übergänge zwischen primärer und posttraumatischer Schultersteife sind hier fließend.

Tab. 1 zeigt die Unterschiede zwischen der primären und der sekundären Schultersteife im Überblick.

\section{Postoperativ}

Auch nach Schulteroperationen kann es zur Entwicklung einer Schultersteife kommen. Nach offenen Eingriffen erklärt sich dies durch Narbenbildung, insbesondere bei begleitender langer Ruhigstellung des Gelenks oder schmerzbedingter übermäßiger Schonung. Ebenso kann sich aber eine Steifigkeit auch nach arthroskopischen Operationen entwickeln, wobei nicht zwingend ein $\mathrm{Zu}$ sammenhang mit der Größe oder Dauer des Eingriffs besteht. Bei arthroskopischen Rotatorenmanschettennähten oder Kalkentfernungen muss z.B. mit einer Häufigkeit von ca. 2-5\% mit dieser Komplikation gerechnet werden. Bemerkbar macht sie sich durch anhaltende bzw. zunehmende Schmerzen auch 4-6 Wochen postoperativ, vor allem auch Ruhe- und nächtliche Schmerzen, sowie eine passiv eingeschränkte Beweglichkeit mit schmerzhaftem Anschlag. Arthroskopisch zeigt sich bei einer Revision zunächst eine ausgeprägte Synovitis mit Rötung, später dann mit Übergang zu einer ligamentären und kapsulären Fibrodysplasie, analog zur frozen shoulder.

- Cave: Eine übermäßig aggressive Physiotherapie ohne Respektierung der Schmerzgrenze oder auch zu frühe Kräftigungsübungen scheinen diese Komplikation zu fördern.

Therapeutisch kommen physio- und manualtherapeutische Behandlungen im schmerzarmen Bereich mit Dehnübungen und ohne Belastung oder Widerstandsübungen zum Einsatz. Auch eine intraartikuläre oder orale Kortisontherapie ist möglich, allerdings mit weniger guten Erfolgschancen als bei der primären Schultersteife im frühen Stadium. Das Vorhandensein von Ruheschmerzen als Zeichen eines entzündlichen Vorgangs kann als Hinweis auf das eventuelle Ansprechen auf eine Kortisontherapie interpretiert werden, wohingegen ausschließlich bewegungsabhängige Beschwerden erfahrungsgemäß weniger gut ansprechen.
Tabelle 1

\begin{tabular}{|c|c|c|}
\hline & primäre Schultersteife & sekundäre Schultersteife \\
\hline Verlauf & $\begin{array}{l}\text { typischer phasenhafter } \\
\text { Verlauf }\end{array}$ & Phasen nicht immer abgrenzbar \\
\hline Selbstheilungstendenz & gut & gering \\
\hline Dauer & $\begin{array}{l}6 \text { Monate bis } 2 \text { Jahre } \\
\text { (selten auch länger) }\end{array}$ & ungewiss \\
\hline
\end{tabular}

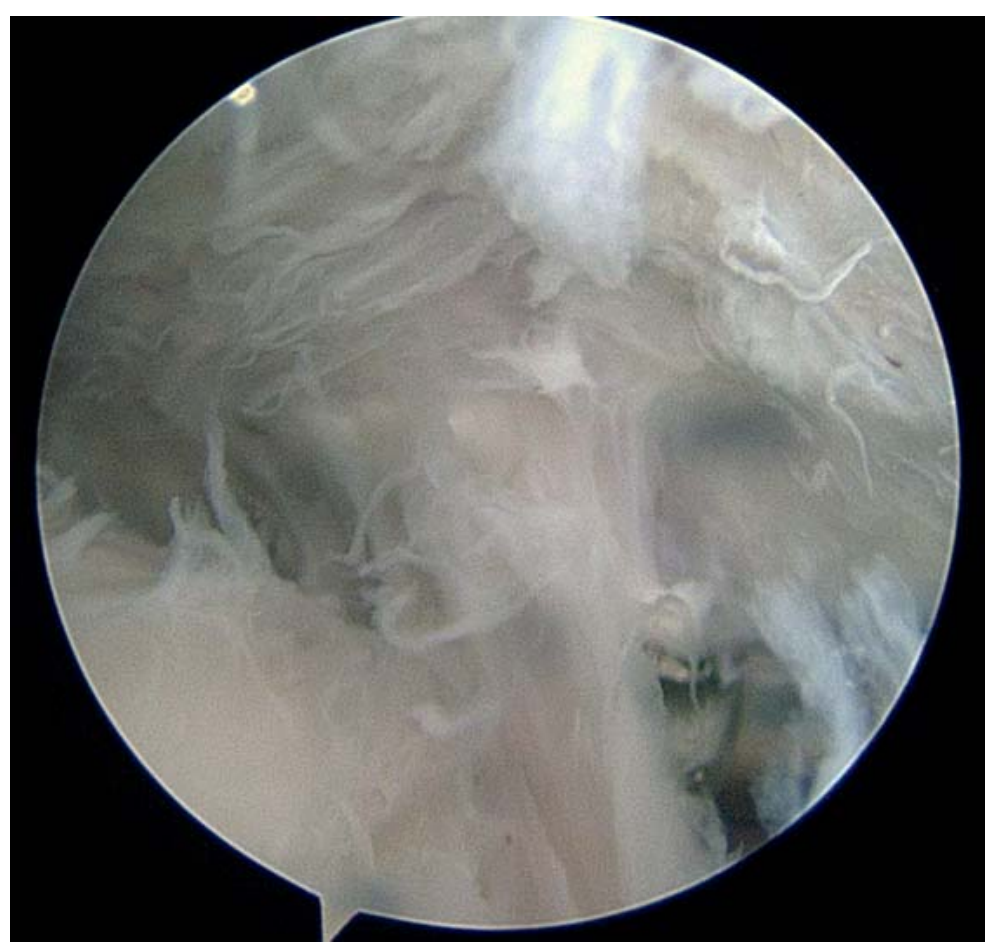

Abb. 2 - Endoskopischer Blick über ein dorsales Standardportal in den subakromialen Gleitraum einer rechten Schulter, unten links die Oberfläche der Rotatorenmanschette/ Supraspinatussehne nach Mini-open-Naht ohne Reruptur, aber mit ausgeprägten narbigen Bridensträngen zur Unterfläche des M. deltoideus (oben im Bild), von lateral (rechts) Beginn der Adhäsiolyse mit dem Shaver.

Nach Rotatorenmanschettennähten finden sich als Ursache von Bewegungseinschränkungen auch rein narbige Veränderungen ohne synovitische/kapsulitische Komponente; eine eindeutige Differenzierung ist meist nur intraoperativ möglich (Abb. 2). Bei fehlenden Fortschritten ist bei beiden Formen im Verlauf die Indikation zur Rearthroskopie zu stellen, bei ca. 80\% der Patienten sollte sich damit eine Verbesserung von Beweglichkeit und Schmerzen erreichen lassen. 
Bei anhaltender Bewegungseinschränkung nach arthroskopischen Stabilisierungen ist die Unterscheidung zwischen Schultersteife durch exzessive Narbenbildung oder Fibrosierung der ventralen Kapsel und einer operationstechnisch bedingten übermäßigen vorderen Kapselraffung im Rahmen der Labrumrefixierung ebenfalls schwierig. Beide Formen können in Abhängigkeit vom Verlauf durch eine arthroskopische Arthrolyse bzw. Kapsulotomie behandelt werden.

Des Weiteren werden auch nach Operationen ohne Beteiligung des Schultergelenks wie bei Eingriffen am Gehirn, an Thorax und Abdomen und bei Mamma-OPs postoperative Schultersteifen beschrieben, ohne dass die genauen Zusammenhänge bekannt wären. Teils konnten Risikofaktoren identifiziert werden. Auch nach SchädelHirn-Traumata und -Blutungen besteht eine erhöhte Inzidenz von Schultersteifen.

Inaktivität und Nichtgebrauch spielen eine wichtige Rolle, sodass bei all diesen Patienten frühe passive Bewegungen und frühe eigene Übungen zur Prävention angezeigt sind.

Ferner sind medikamentös induzierte Formen der Schultersteife - durch Einnahme von Proteaseinhibitoren wie Virostatika und Metalloproteasen - beschrieben.

\section{Folge anderer Schultererkrankungen}

Eine weitere sekundäre Form besteht in der Entwicklung einer Schultersteife im Rahmen einer primär anderen Schultergelenkerkrankung. So kann ein einfaches Subakromialsyndrom mit Bursitis oder ein Rotatorenmanschettendefekt zu einer sekundären Schultersteife führen. Der Leitbefund ist wiederum eine eingeschränkte passive Beweglichkeit, die nicht zu den primären Krankheitsbildern gehört.

Die Therapie sollte sich dann in erster Linie nach der Schultersteife richten, ähnlich wie bei den anderen sekundären Formen und auch der idiopathischen Kapsulitis; zunächst mit konservativen, ggf. im Verlauf auch mit operativen Mitteln.

\section{Diagnostisches Vorgehen}

\author{
Anamnese \\ - Zur Unterscheidung der primären und der sekundären \\ Schultersteife ist die Anamnese das wichtigste Instru- \\ ment.
}

Die primäre Form und damit die klassische adhäsive Kapsulitis beginnt oft plötzlich und rasch zunehmend mit Schmerzen im Schulterbereich. In der Initialphase stehen die Schmerzen im Vordergrund, diese werden als heftige, oft messerstichartig einschießende Schmerzen tief im Gelenk verspürt, die sich bei Bewegung verstärken, aber auch nachts und in Ruhe ein hohes Ausmaß annehmen und zu Schlafstörungen führen können. Die Bewegungseinschränkung wird in diesem Stadium weniger wahrgenommen.

Bei meist unvermindert anhaltenden Schmerzen wird diese jedoch im 2. Stadium immer deutlicher. Zunächst ist meist die Rotation betroffen, einfache Außenrotationsbewegungen wie der Griff nach einer Türklinke oder nach einem Gegenstand auf der Rückbank eines PKWs sind mit massiven Schmerzen verbunden und nicht mehr möglich. Wenn die Innenrotation ebenfalls betroffen ist, kann der Schürzengriff nicht mehr durchgeführt werden, Frauen berichten oftmals von der Unmöglichkeit, den $\mathrm{BH}-$ Verschluss zu erreichen.

Im weiteren Verlauf ist dann auch die Flexion und die Abduktion eingeschränkt, verbunden mit Schwierigkeiten bei Tätigkeiten oberhalb der Horizontalen wie Gegenstände aus einem hohen Schrank zu nehmen oder sich die Haare zu kämmen. Im Rahmen der „frozen phase“ lassen die Schmerzen bei Zunahme der Bewegungseinschränkung langsam nach. Bei rückläufigen Schmerzen können sich viele Patienten an die Einschränkungen anpassen und kommen damit zurecht, je nach Ausmaß und zeitlicher Entwicklung wird aber auch in diesem Stadium oft der Arzt konsultiert.

\section{Klinik}

Die Schultersteife ist eine klinische Diagnose.

Die Diagnose bzw. zumindest der dringende Verdacht auf eine Schultersteife kann bereits bei der klinischen Untersuchung gestellt werden. Die Schmerzen werden von den Patienten oft dorsal am Schultergelenk lokalisiert, aber auch laterale, ventrale oder diffuse Schulterschmerzen sind möglich. Typisch ist auch eine Ausstrahlung in den 
Oberarm oder bis zum Ellenbogen. Muskuläre Beschwerden im Bereich des Schulterblatts können ebenfalls vorhanden sein. Es kann eine schmerzbedingte Schonhaltung mit Schulterhochstand der betroffenen Seite vorliegen.

Das auffälligste inspektorische Merkmal ist der gestörte skapulothorakale Rhythmus.

Bewegungen im Schultergelenk sind immer Kombinationen der verschiedenen Teilgelenke des Schultergürtels, also von glenohumeralem und akromioklavikulärem Gelenk sowie den funktionell einem Gelenk entsprechenden Gleiträumen zwischen Rotatorenmanschette und akromiohumeralem Bogen bzw. zwischen der Skapula und dem Thorax. Eine Elevations- oder Abduktionsbewegung findet zu ca. $1 / 3$ im skapulothorakalen Gleitraum und $\mathrm{zu}^{2} / 3$ zwischen Humerus und Skapula statt, wobei zu Beginn der Bewegung aus der Neutralstellung heraus der glenohumerale Anteil überwiegt.

Bei der Betrachtung des Schultergürtels beim entkleideten Patienten von dorsal und der Aufforderung zur gleichzeitigen Abduktion beider Arme kommt es auf der erkrankten Seite bei der Schultersteife aufgrund der eingeschränkten glenohumeralen Beweglichkeit bereits von Beginn an zu einer Mitbewegung der Skapula (Abb. 3). Alternativ kann die skapulohumerale Beweglichkeit getestet werden, während die Skapula mit einer Hand fixiert und dann das Ausmaß der möglichen Abduktion im Seitenvergleich untersucht wird (auf der erkrankten Seite oft nur $30^{\circ}$ ).

Auch eine Bewegungseinschränkung im Gleitraum der Rotatorenmanschette, z. B. durch postoperative Narbenbildung führt zu diesem gestörten Skapularhythmus, allerdings meist weniger ausgeprägt. Eine Einschränkung der Elevation/Abduktion durch eine fehlende Beweglichkeit im skapulothorakalen Gleitraum bei normal beweglichem Glenohumeralgelenk ist hingegen eine Rarität und kann z. B. nach ausgedehnten Skapulakorpusfrakturen beobachtet werden.

Bei der Palpation findet sich oft ein Druckschmerz über dem AC-Gelenk.

Beim Vollbild einer Schultersteife ist dies als Folge der eingeschränkten glenohumeralen Beweglichkeit und der durch den gestörten Bewegungsablauf vermehrten Druckbelastung des ACG und nicht als eigene AC-bedingte Erkrankung zu sehen. Außerdem können - teils abhängig von der zugrunde liegenden Pathologie -

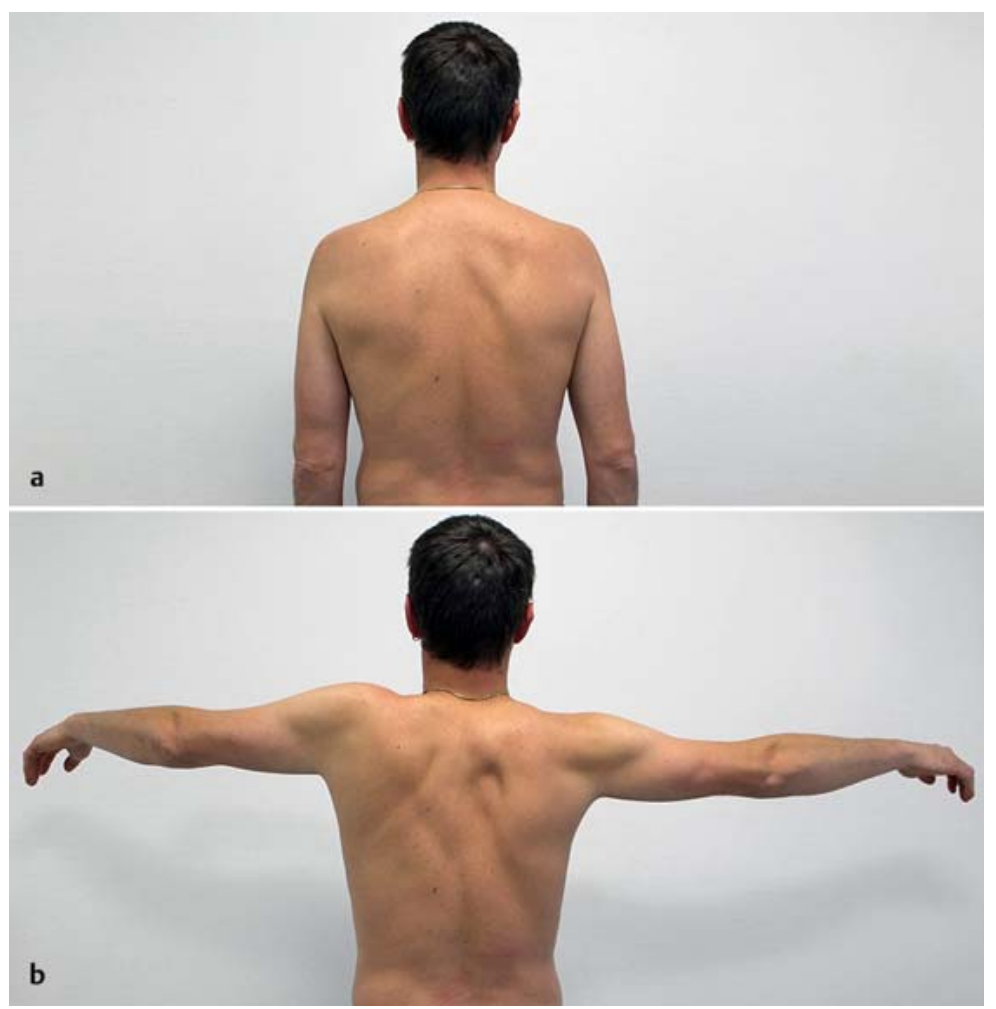

Abb. 3 - 43-jähriger Patient mit Schultersteife links und gestörtem skapulothorakalem Rhythmus. a Ausgangsstellung. b Abduktion fast ausschließlich durch Rotation der Skapula bei eingeschränkter glenohumeraler Beweglichkeit.

Druckschmerzen am ventralen Humeruskopf oder am SSP-Ansatz verspürt werden.

Zur Diagnose einer Schultersteife gehört weiterhin eine Einschränkung der aktiven und passiven Beweglichkeit des Schultergelenks, verglichen mit der gesunden Gegenseite bzw. den alters- und geschlechtsspezifischen Durchschnittswerten. Nach der Neutral-Null-Methode werden insbesondere Flexion und Abduktion sowie Außen- und Innenrotation beurteilt. Retroversion und Adduktion sind am Schultergelenk von untergeordneter Bedeutung.

- Die adhäsive Kapsulitis beginnt typischerweise mit einer Verminderung der Außenrotation, gefolgt von einer Abnahme der Abduktion bzw. auch Flexion und dann der Innenrotation. Dies wird auch als Kapselmuster nach Cyriax bezeichnet.

Nicht ungewöhnlich sind Werte von $0^{\circ}$ Außenrotation (oder selbst negativen Werten bei Unmöglichkeit des Erreichens der Neutralstellung) bei $50^{\circ}$ oder mehr der Gegenseite. Der normale Befund einer mit zunehmender 
Flexion geringeren Außenrotationsfähigkeit kann verstärkt beobachtet werden, sodass der Arm bei zunehmender Flexion praktisch in eine Innenrotation gezwungen wird. Dies scheint vor allem bei stark fibrosiertem MGHL als Ursache der Steifigkeit der Fall zu sein.

Sehr selten kann eine (alte) SSC-Sehnenruptur mit vermehrter Außenrotationsfähigkeit eine beginnende Steife der Gegenseite vortäuschen und muss differenzialdiagnostisch ausgeschlossen werden.

Eine reine Begrenzung der aktiven Beweglichkeit mit passiv freiem Bewegungsumfang wie bei Rotatorenmanschettendefekten sollte durch die klinische Untersuchung unterschieden werden. Bei der Schultersteife findet sich ein - je nach Stadium der Erkrankung mehr oder weniger schmerzhafter - auf kurzer Strecke federnder und „fester Anschlag“ bei der Überprüfung der passiven Beweglichkeit, der Anschlag ist in einer frühen Krankheitsphase eher weich, später eher hart. Teils können jedoch ausgeprägte Schmerzen die Aussagekraft einer Untersuchung einschränken, sodass keine Feststellung getroffen werden kann, ob das Gelenk passiv frei beweglich ist. Eine Unterscheidung, ob es sich um eine schmerzbedingte oder „anatomisch bedingte“ Bewegungseinschränkung handelt, ist dann nur im Rahmen einer Narkoseuntersuchung unter Ausschaltung der Schmerzen möglich.

Aufgrund der begleitenden Schmerzen ist die Aussagekraft der üblichen Funktionstests bezüglich eines Impingements oder der Rotatorenmanschettentests begrenzt. Die Impingement-Tests nach Neer und Hawkins werden bei einer Kapsulitis im Stadium 1 oder 2 immer schmerzhaft und damit positiv sein, ohne dass sie als Zeichen eines Engpasssyndroms interpretiert werden sollten.

\section{Differenzialdiagnostik}

Differenzialdiagnosen sind vor allem die primäre Omarthrose, die zwar ebenfalls mit einer passiven Bewegungseinschränkung einhergeht, aber radiologisch abgegrenzt werden kann und die nicht zu den sekundären Steifigkeiten gezählt wird, außerdem Monarthritiden aus dem rheumatischen Formenkreis, die anamnestisch und laborchemisch unterschieden werden können, und letztlich Gelenkinfekte, die über MRT und Keimnachweis (Punktion, operativ) diagnostiziert werden.

Bei postoperativ anhaltenden und übermäßigen Schmerzen ist eine wichtige Differenzialdiagnose noch das chronisch regionale Schmerzsyndrom. Beim CRPS (Morbus
Sudeck) liegen jedoch begleitende dystrophe Störungen des gesamten Armes vor, die nicht nur auf die Schulter beschränkt sind.

\section{Bildgebung}

Per Definition ist bei der primären Schultersteife die bildgebende Diagnostik mit Röntgen unauffällig. Röntgenaufnahmen in 2 (true a.-p. und axial) bzw. 3 Ebenen (mit Y-Aufnahme) sind in jedem Fall anzufertigen, da eine der wichtigsten Differenzialdiagnosen die primäre Omarthrose ist, die sich anamnestisch und klinisch sehr ähnlich wie eine frozen shoulder präsentieren kann. Eine seltenere Differenzialdiagnose mit ähnlicher Bewegungseinschränkung nach einem Trauma ist eine verhakte hintere Luxation, die sich ebenfalls nativradiologisch oder spätestens in der Schnittbilddiagnostik abgrenzen lässt. Radiologisch können zusätzlich knöchern bedingte Fehlstellungen oder Dysplasien ausgeschlossen werden, ebenso eine begleitende bzw. ursächliche Kalkschulter. In ausgeprägten chronischen Situationen ist eine Osteopenie bzw. Inaktivitätsosteoporose erkennbar, dann ist auch die Skelettszintigrafie positiv.

Auch die Sonografie dient bei der klassischen Kapsulitis nicht zur Bestätigung der Diagnose, sondern dem Ausschluss der wichtigen Differenzialdiagnosen wie Rotatorenmanschettendefekten und Omarthrose. Erstere sind direkt sichtbar, Letztere zeigen sich z.B. durch nachweisbare Osteophyten und Gelenkerguss.

Die MRT kann zwar einige Hinweise auf die Diagnose liefern, aber sichere Kriterien existieren nicht. Zur Diagnostik der Kapsulitis in der Magnetresonanztomografie existieren mehrere Studien. Teils kann eine vermehrte Flüssigkeitsansammlung und damit Signalanhebung in den ventralen kapsuloligamentären Strukturen und im Rotatorenintervall gesehen werden (Abb.4). Eine Verdickung des Lig. coracoacromiale und der Kapsel im Bereich des Intervalls, peritendinöse Veränderungen der langen Bizepssehne, eine Obliteration des Fettanteils zwischen Akromion und Lig. coracoacromiale sowie Veränderungen des Recessus capsularis mit reduziertem Volumen können weitere - ebenfalls unspezifische MR-tomografische Zeichen einer adhäsiven Kapsulitis sein.

Atrophien der Muskulatur der Rotatorenmanschette ohne begleitende Sehnendefekte können in ausgeprägten Stadien vorhanden sein. Hierbei muss aber auch an die seltene neuralgische Schulteramyotrophie gedacht werden. Insgesamt liegt bei der MRT der Vorteil in erster Linie in der Feststellung bzw. dem Ausschluss von Diffe- 

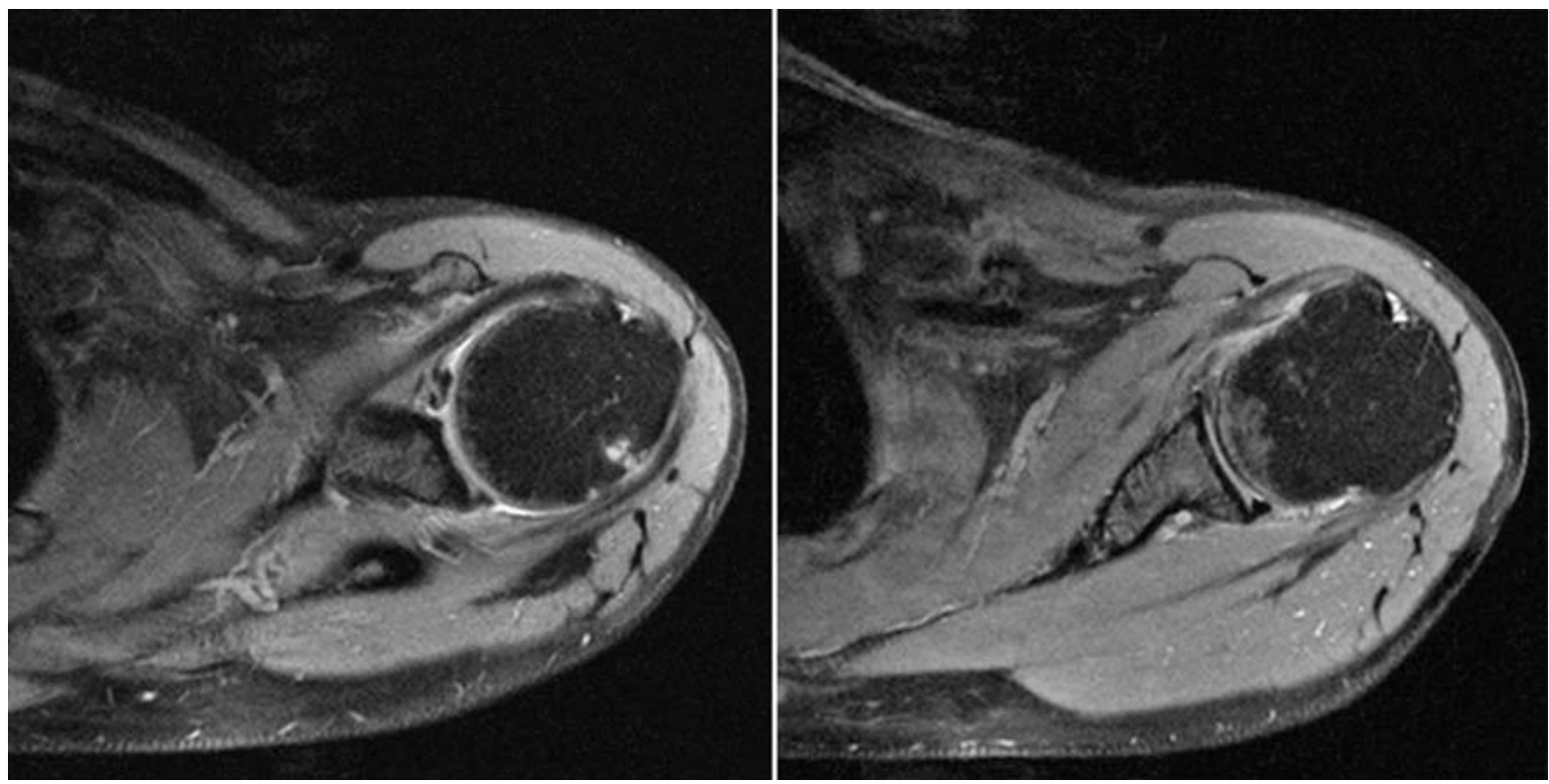

Abb. 4 - Axiale T2-gewichtete MRT-Aufnahmen eines linken Schultergelenks mit primärer Schultersteife und vermehrter Signalanhebung/Flüssigkeitsansammlung in den ventralen und dorsalen kapsuloligamentären Strukturen sowie im Bereich des Rotatorenintervalls.

renzialdiagnosen. Im Rahmen von statischen Bildern eines Gelenks kann selbstverständlich das Ausmaß einer Bewegungseinschränkung als wichtigstes Merkmal der Schultersteife nicht wiedergegeben werden.

Die Computertomografie hat ihren Stellenwert hauptsächlich in der posttraumatischen Situation, z.B. um knöcherne Fehlstellungen nach Frakturen oder die Lage von Implantaten nach Osteosynthesen genau wiederzugeben.

Invasive diagnostische Maßnahmen wie die Arthrografie, die ein reduziertes intraartikuläres Gelenkvolumen und einen verminderten Recessus axillaris nachweisen kann, werden heute nicht mehr eingesetzt.

\section{Therapeutisches Vorgehen}

Die Therapie richtet sich nach der Krankheitsphase bei der primären Schultersteife und berücksichtigt die Ursachen bei der sekundären Form, sie muss wie immer auf die individuellen Verhältnisse des Patienten abgestimmt werden. Der Schweregrad und die Dauer der Schmerzen und der Bewegungseinschränkungen müssen beachtet werden, um eine über- oder untertriebene Therapie zu vermeiden. Das therapeutische Spektrum reicht von abwartendem Verhalten ohne spezifische Maßnahmen (,skillful neglect“) über Schmerzmedikation mit NSAID, Kortisoninjektionen und orale Kortisontherapie für unterschiedlich lange Zeiträume, Krankengymnastik, manuelle Therapie und eigenständige Übungen bis hin zu invasiveren Maßnahmen wie geschlossener Mobilisierung oder Kapseldistension und operativer Therapie mit offenen und arthroskopischen Verfahren.

- Trotz zunehmendem Bekanntheitsgrad und verbessertem Verständnis der Erkrankung finden sich überraschend oft Patienten mit bereits längerer Krankheitsgeschichte und mehreren Arztkontakten, bei denen die korrekte Diagnose noch nicht gestellt wurde.

Wichtig ist in jedem Fall eine ausführliche Aufklärung des Patienten über die Art der Erkrankung, die zumindest bei der primären Form trotz des teilweise langen Verlaufs gutartige Prognose und über mögliche Therapieoptionen. Eine definierte Diagnose und Informationen über Ursachen und Aussichten sind für viele Patienten für die Akzeptanz und Bewältigung bereits äußerst hilfreich.

\section{Konservative Therapie}

Ziel der Behandlung ist entsprechend der Hauptsymptomatik eine rasche Schmerzreduktion sowie eine Verbesserung der Beweglichkeit mit Wiederherstellung einer normalen Schulterfunktion. Es sollte die Verkürzung der teils langen Krankheitsverläufe angestrebt werden. Für eine Vielzahl von Maßnahmen - wie Wärme und Kälte, Schonung und Aktivität, Analgetika und NSAID, Massage, Chiropraxis, Triggerpunktbehandlung, Iontophorese, extrakorporale Stoßellentherapie, N.-suprascapularisBlockaden, Ultraschall und TENS sowie Röntgenreiz- 


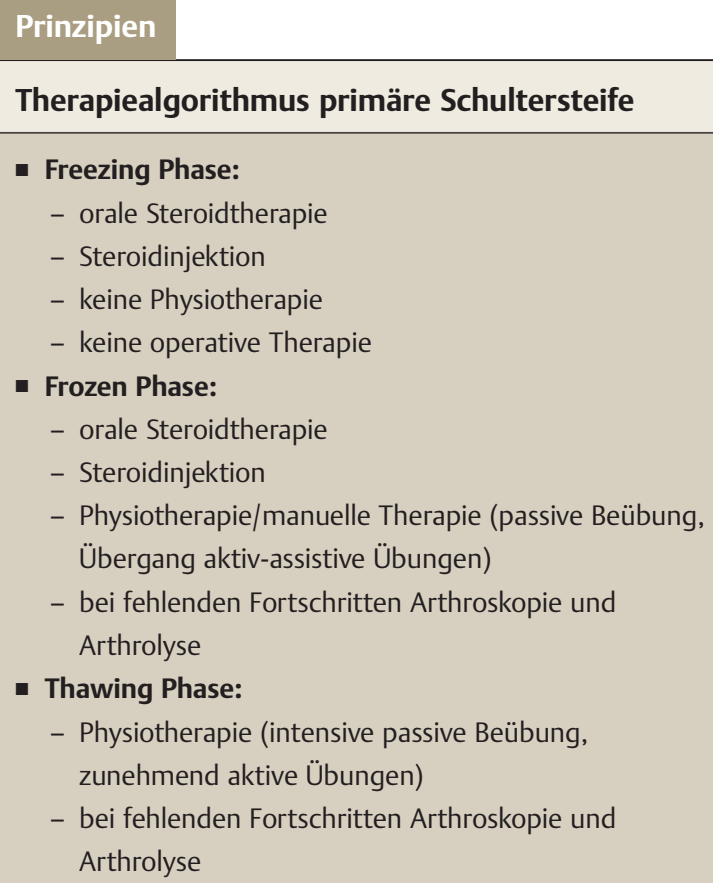

bestrahlung - wurde eine geringe oder moderate positive Beeinflussung der Schultersteife in einzelnen Studien nachgewiesen, die sich in ihren Wirksamkeiten teils kaum unterscheiden. Wenn man den oft selbstlimitierenden Charakter der Erkrankung bedenkt, können diese Maßnahmen als Unterstützung geeignet sein. Nur bei schwerem und prolongiertem Verlauf mit inakzeptablen Beschwerden sind aggressivere Therapieoptionen wie die Mobilisierung oder operative Verfahren angezeigt.

Sowohl für die Gabe von Analgetika als auch von NSAID wurden positive Effekte auf den Verlauf der Erkrankung nachgewiesen. Diese verbesserten sich, wenn die Einnahme mit anderen Maßnahmen kombiniert wurde. In der akuten „freezing phase“, aber auch im zweiten Stadium und bei der sekundären Form wird subjektiv jedoch sehr oft keinerlei Schmerzlinderung verspürt. Selbst die zusätzliche Gabe von Opioiden ist dann nicht ausreichend für eine akzeptable Schmerzreduktion.

\section{- Steroidtherapie}

Orale Therapie. Aufgrund der hohen antiinflammatorischen Potenz von Kortison ist dieses vor allem in den entzündlichen Stadien 1 und 2 der Kapsulitis häufig gut wirksam. Es existieren unterschiedliche Schemata zur oralen Gabe von Kortison, am meisten findet Decortin $\mathrm{H}$ Verwendung. Es wird mit einer höheren Dosis begonnen und schrittweise reduziert. Die Einnahme sollte morgens erfolgen (zirkadianer Rhythmus der körpereigenen Kortisonproduktion) und mit einem Protonenpumpenhemmer kombiniert werden. Von Habermeyer (2003) wurde ein Schema über 35 Tage etabliert, später wurde eine Einnahme über 20 Tage als gleich wirksam beschrieben. Bei korrekter Indikationsstellung ist mit einem guten Ansprechen bei 70-80\% der Patienten zu rechnen. Es kann einen bis mehrere Tage dauern, bis die antientzündliche und damit auch schmerzreduzierende Wirkung verspürt wird.

Während der Einnahme wird auf physiotherapeutische Behandlung verzichtet. Es werden lediglich Bewegungen im schmerzarmen Bereich empfohlen. Ist es nach Beendigung der Kortisoneinnahme zu einer deutlichen Schmerzreduktion und manchmal auch bereits Bewegungsverbesserung gekommen, kann dann die Krankengymnastik, die zuvor oft schmerzbedingt nicht möglich war, erneut begonnen werden.

Im späten Stadium 2 sowie im Stadium 3 der frozen shoulder sowie bei der sekundären Schultersteife ist die Kortisontherapie weit weniger erfolgreich, auch bei einem Teil der Patienten mit primärer Schultersteife im frühen und mittleren Stadium lässt sich keine Verbesserung erzielen. Außerdem gibt es Patienten, die nach initial guter Schmerzreduktion über wieder zunehmende Symptome während der Reduzierung der Dosis klagen. Eine dauerhafte Einnahme der Präparate wird wegen der unerwünschten Arzneimittelwirkungen von Kortison jedoch nicht empfohlen. Die möglichen Nebenwirkungen der kurzfristigen Gabe von Decortin sind mit Magenbeschwerden, Hypertonie, Tachykardien, Hyperglykämien, Kopfschmerzen, Schlaflosigkeit, Stimmungsschwankungen und Wassereinlagerungen zwar vielfältig, aber zumeist mild ausgeprägt und reversibel.

Bei bekanntem Diabetes mellitus besteht eine Kontraindikation für eine orale Kortikoidmedikation bzw. diese ist äußerst kritisch zu bewerten. Nur in Ausnahmefällen kann nach Absprache mit dem behandelnden Diabetologen eine solche Therapie durchgeführt werden, wenn von Patientenseite ein hoher Leidensdruck und entsprechender Wunsch besteht. Alternativ kann in dieser Situation auch eine intraartikuläre Injektion mit einem Steroid erfolgen.

Injektionstherapie. Injektionen haben in einigen Studien ähnlich gute Erfolge - vor allem bezüglich Schmerzreduktion, weniger in Bezug auf verbesserte Beweglichkeit - wie die orale Gabe (oder auch die Physiotherapie) erzielen können. Sie können jedoch nur mit erhöhtem 
logistischem Aufwand durchgeführt werden. In der Regel sind eine Bildwandlerkontrolle und eine Kontrastmittelgabe zur Überprüfung der korrekten intraartikulären Applikation erforderlich. Außerdem besteht immer das wenn auch geringe - Risiko eines iatrogenen Gelenkinfekts. Aufgrund der nachgewiesenen negativen Auswirkungen von Kortikosteroiden auf Sehnen- und hyalines Knorpelgewebe dürfen Injektionen nicht zu oft wiederholt werden. Subakromiale Injektionen sind bei der frozen shoulder mit ihrer nicht primär im Gleitraum lokalisierten Pathologie sinnlos.

\section{- Physiotherapie}

> Der entscheidende Baustein der konservativen Therapie in der chronischen Phase ist die Physiotherapie inklusive Anleitung und Durchführung von eigenständigen Übungen.

$\mathrm{Zu}$ beachten ist, dass in einer frühen Krankheitsphase die Physiotherapie kontraindiziert ist, da das wiederholte Bewegen des Schultergelenks an die tolerablen Grenzen eine Reizung der Kapsel verursacht, die eine Verschlechterung der Symptomatik mit sich bringt. Patienten in dieser Situation brechen die Krankengymnastik oft wegen zunehmender Schmerzen selbstständig ab. In der 2. und 3. Phase der primären und bei der sekundären Schultersteife ist eine schmerzadaptierte Physiotherapie mit sanften bis festen passiven Dehnübungen in allen Bewegungsebenen jedoch absolut sinnvoll und mit guten Effekten belegt.

Die Eigeninitiative des Patienten sollte gefördert werden. Erlernte Übungen müssen regelmäßig zu Hause wiederholt werden. Eine häufige, am besten mehrfach tägliche Durchführung ist besser als wenige, aber aggressivere Dehnungen. Von Matsen bzw. der Physiotherapeutin Jackins (1994) ist ein nach ihr benanntes Heimprogramm mit Dehnübungen etabliert worden. Der wiederholte Reiz einer mechanischen Dehnung auf das fibrosierte Kapselgewebe soll als entscheidender Auslöser für eine Anpassung des Gewebes an diesen Reiz mit entsprechender Relaxierung der Fasern wirken.

Auch die manuelle Therapie mit insbesondere posteriorem Gleiten und passiver Mobilisierung kann mit positiven Auswirkungen angewendet werden.

\section{Invasive und operative Therapie}

\section{narkosemobilisierung}

Die Narkosemobilisierung - oder das Brisement forcé ist mit Problemen verbunden und sollte als alleinige Maßnahme heute nicht mehr zur Anwendung kommen.

Beim Patienten in Narkose oder Plexusanästhesie und Rücken- oder Beach-Chair-Lagerung wird bei der Mobilisierung die Skapula fixiert und über ein Durchbewegen des Oberarms am kurzen Hebel eine freie Beweglichkeit des Schultergelenks mit hör- und fühlbarer Zerreißung der fibrosierten Gelenkkapsel herbeigeführt. Die Reihenfolge beim Durchbewegen, zunächst Flexion, dann Abduktion und zuletzt Außen- und Innenrotation, muss eingehalten werden. Auf eine aggressive oder ruckartige Mobilisierung muss verzichtet werden.

Weder ist jedoch eine genaue Abklärung der vorliegenden intraartikulären und subakromialen pathologischen Veränderungen möglich noch eine gezielte Therapie derselben. Es kann vielmehr zu einem wahllosen Zerreißen von Weichteilgewebe mit dem Risiko der Schädigung auch relevanter Strukturen wie z.B. der (evtl. vorgeschädigten) Rotatorenmanschette oder des Bizepsankers kommen. Bei Mobilisierung in die Rotation besteht außerdem eine hohe Gefahr (vor allem bei begleitender Osteoporose oder vorangegangener Fraktur) auch für knöcherne Verletzungen - typischerweise im Sinne einer Spiralfraktur des proximalen Humerus -, die dann die Prognose extrem verschlechtern. Die geschlossene Mobilisierung kann jedoch maßvoll in Kombination mit einer arthroskopischen Arthrolyse eingesetzt werden.

\section{Distensionsarthrografie}

Die radiologisch kontrollierte Punktion des Schultergelenks mit anschließendem Auffüllen des Gelenks und zuletzt einer durch den Überdruck herbeigeführten Zerreißung der Kapsel wird ebenfalls kaum noch durchgeführt. Die Intervention ist beim wachen Patienten auch bei Verwendung von Lokalanästhetika überaus schmerzhaft, und eine gezielte Therapie ist wie bei der geschlossenen Mobilisierung nicht möglich. Das Gewebe reißt an der Stelle des geringsten Widerstands, und die am meisten verdickten Kapselanteile werden nicht adressiert.

\section{- Offene Arthrolyse}

Die offene Arthrolyse ist heute nicht mehr indiziert, da der Flurschaden den Nutzen überwiegt. 
Beim offenen Eingriff können über einen deltoideopektoralen Zugang die extraartikulären Anteile der Schulter erreicht werden. Der gesamte subakromiale/subdeltoidale Gleitraum kann scharf unter Sicht oder stumpf-digital mobilisiert werden. Die intraartikulären Probleme können jedoch kaum behandelt werden. Auch nach Eröffnen des Rotatorenintervalls ist der Gelenkraum nur sehr eingeschränkt zugänglich, die dorsale Kapsel kann überhaupt nicht inzidiert oder gelöst werden. Die Verlängerung einer kontrakten SSC-Sehne wird heute (zu Recht) überhaupt nicht mehr durchgeführt, da hohe Risiken wie SSC-Insuffizienz und erneute Kontraktur drohen. Die erforderliche postoperative Ruhigstellung nach SSCSehnennaht ist für das Nachbehandlungskonzept kontraproduktiv.

Die Übersicht im Gelenk und die Behandlungsmöglichkeiten sind arthroskopisch definitiv besser, dem versierten Operateur ist auch der subakromiale Gleitraum ggf. unter Verwendung zusätzlicher Portale vollständig zugänglich. Bei der offenen Arthrolyse sind mehr Narbenbildungen und Adhäsionen als bei einem arthroskopischen Eingriff zu erwarten, sodass das Risiko einer erneuten Bewegungseinschränkung bzw. einer insgesamt kaum verbesserten Beweglichkeit besteht. Auch verschlechtern die nachgewiesenen höheren Schmerzen bei offenen Operationen die Möglichkeiten einer sofortigen postoperativen Bewegungstherapie und damit die Resultate.

Bei sekundären Steifigkeiten nach proximalen Humerusfrakturen mit der Notwendigkeit einer Plattenentfernung ist die Kombination einer arthroskopischen intraartikulären Arthrolyse mit einer arthroskopischen oder offenen Entfernung des Osteosynthesematerials inklusive subakromialer Adhäsiolyse möglich.

\section{- Arthroskopische Arthrolyse}

> Durch die niedrigen Komplikationsraten und die guten Behandlungsergebnisse hat sich die arthroskopische Arthrolyse bei der Schultersteife in den letzten Jahrzehnten als wichtige Therapieoption und operativer Standard etabliert.

Abhängig von der Form der Schultersteife und der zeitlichen Entwicklung wird je nach Autor zuvor eine erfolglose konservative Therapiedauer von 3-6 Monaten gefordert. In der frühen Phase der frozen shoulder ist eine Operation kontraindiziert.

Das bei der Arthrolyse durchgeführte kapsuläre Release führt zu einer Laxität des Gelenks, eine Instabilität mit Luxationen wird hingegen kaum beobachtet. Eine Ver- besserung bis hin zu vollständig normalen Verhältnissen mit Schmerzfreiheit und vollem Bewegungsumfang kann nach einer Arthroskopie mit guten Chancen in sehr vielen Fällen erreicht werden, mit einer fehlenden Besserung muss allerdings in bis zu 10\% der Patienten gerechnet werden. Bei Diabetikern verbleiben häufiger Restbeschwerden.

Indikation. Die Indikation zur arthroskopischen Arthrolyse wird abhängig vom subjektiven Leidensdruck des Patienten und der Ausprägung von Schmerz und Bewegungseinschränkung gestellt. Der Spontanverlauf, das vorliegende Stadium bei der primären Schultersteife und die mögliche Ätiologie bei der sekundären Form müssen berücksichtigt werden, ebenso die vorhandene Bildgebung. Eine konservative Therapie sollte über mindestens 6 Monate ohne ausreichenden Erfolg durchgeführt worden sein. Von Charles Neer wurde für die frozen shoulder die Einschätzung gegeben, dass lediglich 10\% der Patienten einer operativen Therapie bedürfen und 90\% unter konservativen Maßnahmen einen zufriedenstellenden Verlauf zeigen (Neer 1980).

Wenn für Patienten trotz Kenntnis der Prognose und des zu erwartenden Verlaufs aufgrund der Schmerzen und/ oder der Einschränkungen bei der Arbeit oder im täglichen Leben ein weiteres Abwarten nicht mehr möglich ist, kann alternativ eine Arthrolyse mit deutlicher Verkürzung der Krankheitsdauer angeboten werden.

Ebenso müssen bei fehlender vollständiger Wiederherstellung der Schulterfunktion und anhaltenden Restbeschwerden diese nicht dauerhaft akzeptiert werden. Bei persistierenden Bewegungseinschränkungen von $10-20^{\circ}$ zur gesunden Gegenseite, die nur eine Bewegungsebene betreffen, sollte Zurückhaltung bezüglich einer operativen Therapie empfohlen werden, da eine Verbesserung hier nur schwer zu versprechen ist. Bei darüber hinausgehenden Einschränkungen ( $\mathrm{ab}$ ca. $30^{\circ} \mathrm{im}$ Vergleich zur Gegenseite) einer oder mehrerer Ebenen kann jedoch eine relative OP-Indikation bestehen. Eine Ausnahme bildet die eingeschränkte Innenrotation bei sonst normalem Bewegungsumfang, die auch im Rahmen einer Werferschulter als GIRD (glenohumerales Innenrotationsdefizit) vorkommt und die nicht als klassische sekundäre Schultersteife verstanden wird.

Bei einer primären Kapsulitis in der freezing phase sollte keine operative Therapie durchgeführt werden, da in einem hohen Prozentsatz durch die noch hohe Entzündungsaktivität mit einer raschen Wiedereinsteifung des Gelenks gerechnet werden muss. Im Stadium 2 der frozen shoulder sollte den Patienten vor einer operativen The- 
rapie eine orale Kortisontherapie empfohlen werden, wenn keine Kontraindikationen bestehen.

Bei der sekundären Schultersteife ist die Indikation zur operativen Therapie aufgrund der längeren Verläufe und der schlechteren Ergebnisse unter konservativer Therapie aggressiver zu stellen. Hier wird von den meisten Autoren ein 3-monatiger konservativer Therapieversuch gefordert. Insbesondere nach Rotatorenmanschettennähten kann jedoch die Wiedererlangung der freien Beweglichkeit auch einen längeren Zeitraum in Anspruch nehmen und trotzdem auch ohne Revision erreicht werden. Auch nach operativ versorgten Humeruskopffrakturen ist ein bereits wieder normaler passiver Bewegungsumfang des Schultergelenks nach 3 Monaten insbesondere bei älteren Patienten üblicherweise nicht vorhanden.

- Eine entscheidende Frage an den Patienten ist diejenige nach noch vorhandenen Fortschritten unter krankengymnastischer Behandlung sowie mit eigenständigen (Dehn-)Übungen in den letzten Wochen.

Solange sich subjektiv und objektiv Verbesserungen der Beweglichkeit zeigen, ist eine weitere Normalisierung möglich, der Leidensdruck üblicherweise geringer und ein abwartendes Verhalten gerechtfertigt. Ist hingegen ein über mehrere Wochen gleichbleibendes und für den Patienten subjektiv nicht akzeptables Niveau der Bewegungseinschränkung ohne Besserung trotz Physiotherapie erreicht, besteht Handlungsbedarf.

Die postoperative Schultersteife wird auch in der Schulterendoprothetik als Komplikation beobachtet. Vor allem nach anatomischen Prothesen können passive Bewegungseinschränkungen bei regelrechter Implantation und unauffälligem Röntgenbefund in relevantem Ausmaß verbleiben. Die Übergänge zu geringen und als normal empfundenen postoperativen Limitierungen sind hier nicht genau definiert. Bleibt jedoch eine ausgeprägte Einschränkung über 6-12 Monate nach der Implantation trotz intensiver Übungsbehandlung bestehen, kann auch bei einliegender Prothese eine diagnostische Arthroskopie durchgeführt werden. Zum einen können Differenzialdiagnosen wie sekundäre Rotatorenmanschettendefekte, Infekte und Lockerungen abgeklärt werden, zum anderen können - wie bei anderen postoperativen Schultersteifen auch - eine Kapsulotomie und eine Arthrolyse erfolgen.

Ähnliches gilt für die häufigen Schultersteifen nach proximalen Humerusfrakturen und Plattenosteosynthesen. Bei korrekt einliegenden Implantaten ohne Schraubenüberstand an der Kalotte oder Plattenhochstand mit sekundärem Impingement kann durchaus auf eine Entfernung des Osteosynthesematerials verzichtet werden. Der Verzicht auf eine offene Revision ist atraumatischer und mit Vorteilen für den Patienten wie geringeren Schmerzen und geringerer Rezidivhäufigkeit verbunden. Zusätzlich zur intraartikulären Überprüfung und Kapsulotomie muss hier besonderer Wert auf die subakromiale Adhäsiolyse gelegt werden. Oftmals lässt sich eine deutliche und ausreichende Verbesserung auch bei initial suboptimaler Frakturstellung erreichen, die alternativ nur endoprothetisch versorgt werden könnte.

Durchführung. Wie bei anderen arthroskopischen Schulteroperationen wird der Eingriff in Plexusanästhesie allein oder in Kombination mit einer Allgemeinnarkose durchgeführt. Ein Plexuskatheter verbessert nicht nur die intraoperativen Bedingungen (niedrigerer Blutdruck durch weniger Schmerzen), sondern ist auch für die postoperative Schmerztherapie speziell bei der Schultersteife wichtig.

Die meisten Operateure führen den Eingriff in BeachChair-Position durch. Der Vorteil liegt in den meist gewohnten Verhältnissen und bei der intraoperativ überprüfbaren Beweglichkeit. Es ist jedoch auch eine Seitlagerung möglich. Hier ist durch die Distraktion der Gelenkraum weiter, und wenn erforderlich sind die dorsalen Kapselanteile besser zu erreichen.

Wichtig ist auf jeden Fall vor Beginn der Operation die Untersuchung und Dokumentation des passiven Bewegungsumfangs des Schultergelenks in Narkose unter Schmerzausschaltung. Wie oben erwähnt kann teils erst jetzt eindeutig die Diagnose gestellt bzw. ausgeschlossen werden.

Bei Patienten, die jünger als 40 Jahre sind, findet sich selten eine frozen shoulder. Manche Patienten aus dieser Altersgruppe sind aufgrund von ausgeprägten Schmerzen kaum zu untersuchen, in Narkose zeigt sich dann jedoch eine passiv völlig freie und uneingeschränkte Beweglichkeit.

Bei Operationen aus anderen Indikationen sollte diese Beweglichkeitsüberprüfung ebenfalls immer durchgeführt werden.

Zwischen der Untersuchung des Patienten mit Diagnoseund Indikationsstellung und der anschließenden Operation können mehrere Wochen oder sogar Monate liegen. In diesem Zeitraum kann sich eine sekundäre Schultersteife bei primär noch frei beweglichem Gelenk entwickelt haben. Dies ändert dann das operative Vorgehen 


\section{Schultergürtel und obere Extremität}

entscheidend. Von den meisten Autoren wird z. B. das Vorhandensein einer Schultersteife als Kontraindikation für eine Rotatorenmanschettennaht gesehen. Vorrangig ist dann zunächst die arthroskopische Arthrolyse mit anschließend intensiver Krankengymnastik. In vielen Fällen ist bei rückläufigen Beschwerden dann im Verlauf keine Sehnenrekonstruktion mehr erforderlich; sie kann aber bei anhaltend gutem Bewegungsausmaß und symptomatischem Defekt sekundär (üblicherweise Entscheidung ca. 3 Monate nach Arthrolyse) erfolgen (Abb. 5).

Bei primärer Naht eines Rotatorendefekts trotz begleitender Kapsulitis/Steifigkeit ist zwar mit einer Einheilung der Sehne zu rechnen, eine gute Beweglichkeit jedoch nicht zu erwarten. Der Patient wird mit dem Ergebnis nicht zufrieden sein, und eine Folgeoperation ist unumgänglich.

Durchführung. Standardisiert werden die anatomischen Schulterkonturen und die Portale angezeichnet.

Üblich sind

- ein dorsaler Zugang für die Optik,

- ein anterosuperiores Portal für die intraartikulären Maßnahmen und

- ein laterales Portal für die subakromiale Bursektomie/ ggf. Akromioplastik.

Im Vergleich zur normalen Schulterarthroskopie ist die Anlage des dorsalen Zugangs oft schwieriger. Auf jeden
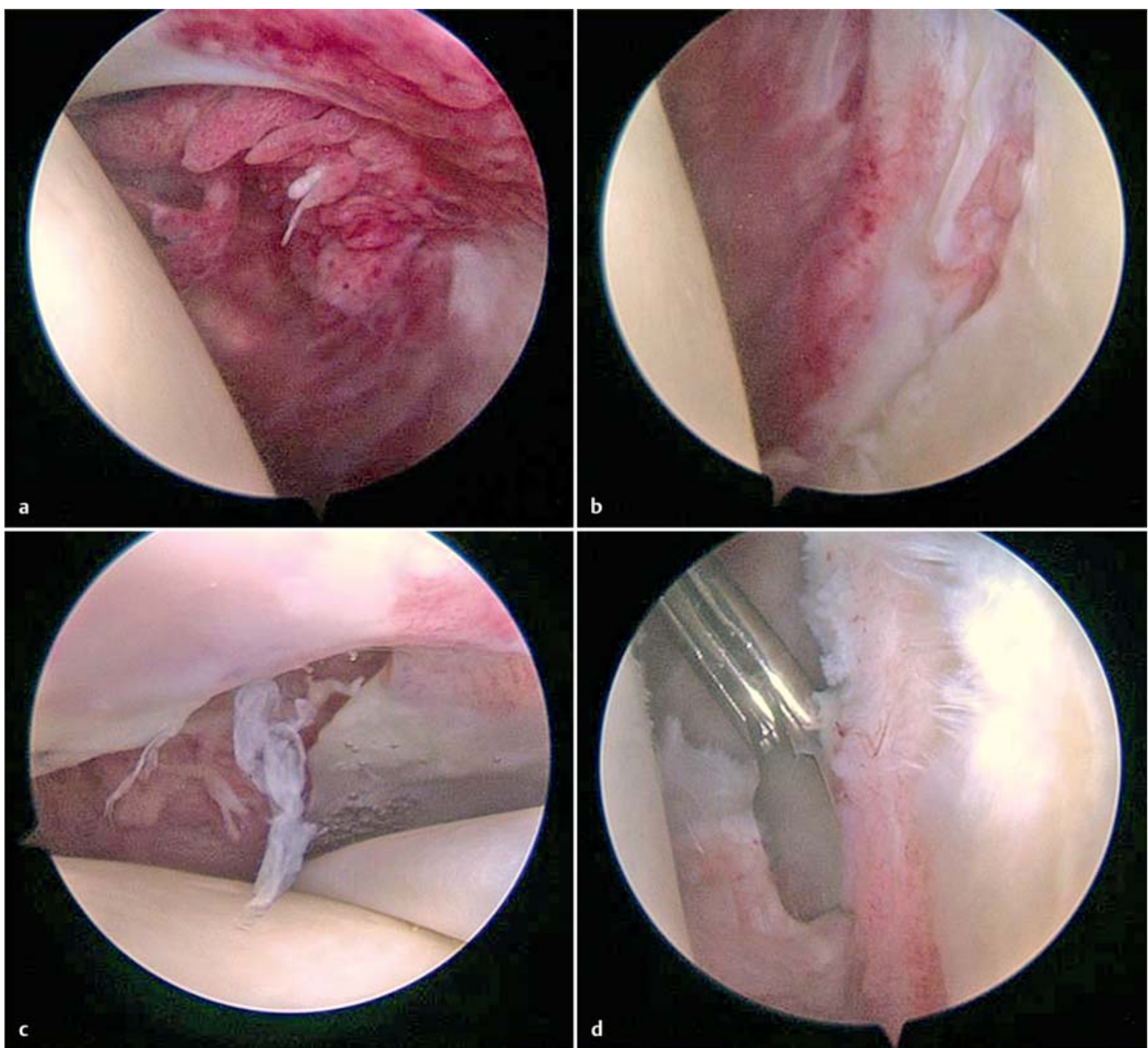

Abb. 5 - Arthroskopische Bilder eines linken Schultergelenks von dorsal. Bei der Narkoseuntersuchung vor der Operation waren bereits eine passiv eingeschränkte Flexion von $100^{\circ}$ und eine Außenrotation von $10^{\circ}$ mit jeweils festem Anschlag aufgefallen. a Typische und auffällige Synovitis im Bereich des Rotatorenintervalls und des Bizepssehnenankers. b Derbes und verdicktes MGHL. c Degenerativer SSP-Defekt. d Therapeutische Arthrolyse/Kapsulotomie (Inzision bzw. Resektion der fibrosierten ventralen Kapsel von kranial nach kaudal parallel zum Labrum mit dem Punch). 
Fall sollte ein stumpfer Trokar verwendet werden.

Durch die Kontraktur ist der Gelenkspalt enger und das Risiko einer Knorpelschädigung beim Eingehen höher. Von Gartsman (2008) wird empfohlen, mit der Trokarspitze nach Perforation von Haut, dorsalem M. deltoideus und Rotatorenmanschette knöchernen Kontakt zu suchen und dann eine Rotation des Oberarmes durchzuführen, bei fühlbarer Bewegung (= Trokarspitze auf dem Humerus) muss die Spitze mehr nach medial gerichtet werden, bei fehlender Bewegung (= Trokarspitze auf der Skapula) mehr nach lateral. Auch die vorherige Punktion mit Auffüllen des Gelenks kann die Anlage des dorsalen Zugangs vereinfachen.

Diagnostischer Rundgang. Nach Etablierung des Kameraportals wird zunächst die Arthroskopie mit diagnostischem Rundgang durchgeführt. Die typischen Befunde bei einer frozen shoulder sind in der Übersicht dargestellt.

\section{Übersicht}

\section{Arthroskopische Befunde einer „frozen shoulder“}

- Synovitis mit Rötung und Zottenbildung im Rotatorenintervall

- Verdickung von SGHL, MGHL, IGHL und ventraler Kapsel

- fehlende Abgrenzbarkeit des kranialen SSC-Sehnenrandes

- Obliteration der Recessus subscapularis und axillaris

- Rötung auch des synovialen Überzugs oberhalb und dorsal des Glenoids sowie im Recessus axillaris
Der Knorpelüberzug von Humeruskopf und Glenoid ist unauffällig, das Labrum kann gerötet und verdickt sein, die lange Bizepssehne zeigt sich intakt, selten mit Adhäsionen zum Rotatorenintervall im Bereich des Eintritts in den Sulcus intertubercularis, und die artikularseitigen Oberflächen von SSP- und ISP-Sehne sind bis auf eine vermehrte Gefäßinjektion oft unter Aussparung des Rotatorenkabels ebenfalls intakt. Im Stadium 3 ist die Synovitis meist weniger und die Verdickung und Fibrosierung des MGHL und der ventralen Kapsel oft deutlicher ausgeprägt als in früheren Stadien.

Therapeutische Maßnahmen. Bei arthroskopischer Bestätigung der frozen shoulder bzw. der sekundären Schultersteife erfolgen dann die therapeutischen Maßnahmen.

Zu Beginn wird über ein anterosuperiores Portal eine partielle Synovektomie mit Entfernung der geröteten Zotten durchgeführt, zur Schmerzreduktion für den Patienten und zur besseren Übersicht für den Operateur. Es folgt die Tenolyse der SSC-Sehne, indem diese vom fibrosierten Bindegewebe ventral und dorsal der Sehne bis medial des vorderen Glenoids befreit wird, ohne das Sehnengewebe selbst zu schädigen (Abb.6).

Das MGHL wird durchtrennt, wodurch sich in der Regel eine sofortige Verbesserung der Außenrotation erreichen lässt. Dann wird anterosuperior (bei 1.00-2.00 Uhr rechte Schulter) die Kapsel parallel zum Labrum ebenfalls inzidiert, ein ggf. verdicktes SGHL medial durchtrennt und ein 5-10 mm breiter Streifen der Kapsel nach kaudal reseziert (Abb. 7). Das Labrum selbst sowie kranial der Bizepsanker dürfen nicht beschädigt werden.

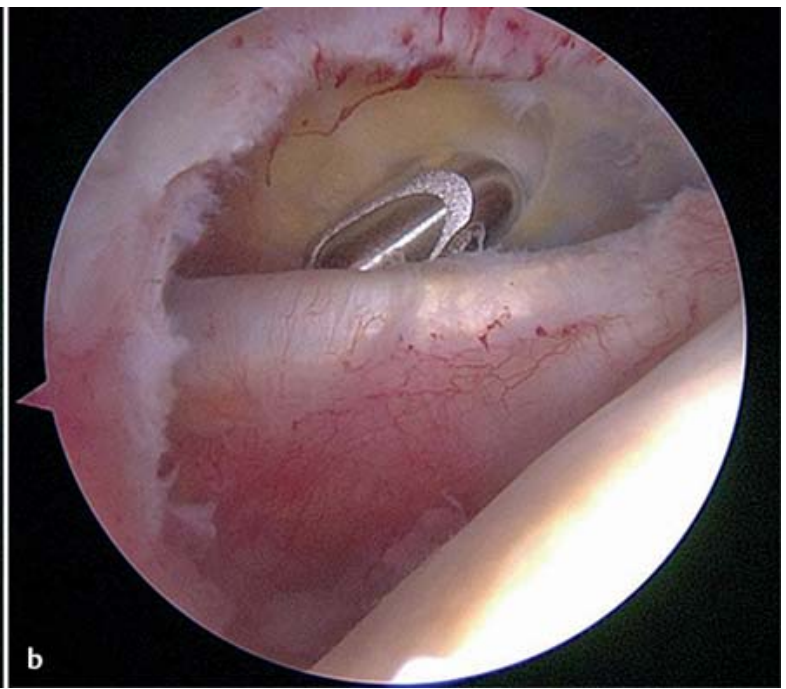

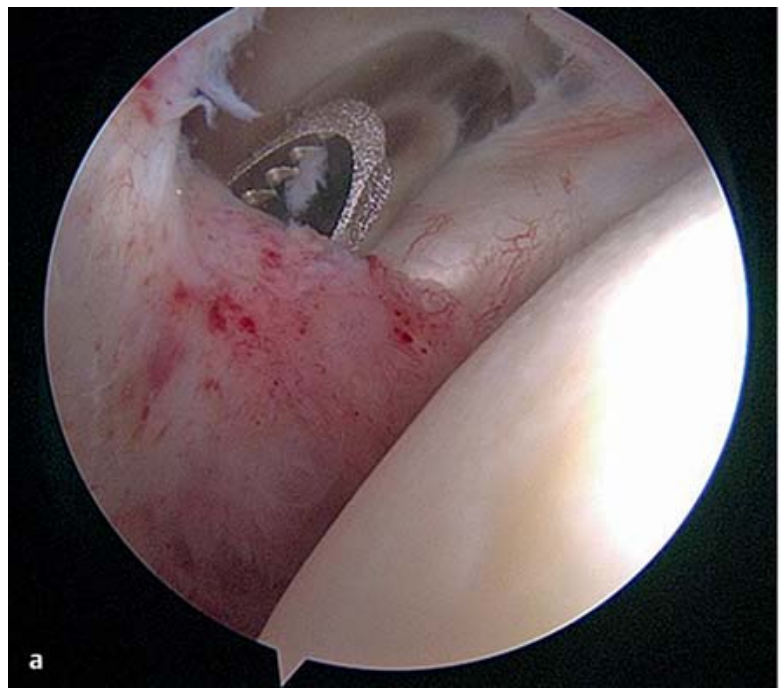

Abb. 6 - Kapsulitis im Stadium 3, Blick von dorsal, rechtes Schultergelenk. a Kaum Synovitis, aber derbe Verdickung des MGHL und auch der Kapsel im Bereich des Rotatorenintervalls. Der Oberrand der SSC-Sehne ist schlecht abgrenzbar. b Nach Inzision und Resektion des MGHL Tenolyse der nun wieder freien SSC-Sehne und Release des Intervalls. 
Abb. 7 - Arthroskopische Arthrolyse bei einer rechten Schulter (Aufsicht von lateral). a Ventrale Kapsulotomie. b Release des Intervalls/Tenolyse der SSC-Sehne (Arbeitskanülen optional).

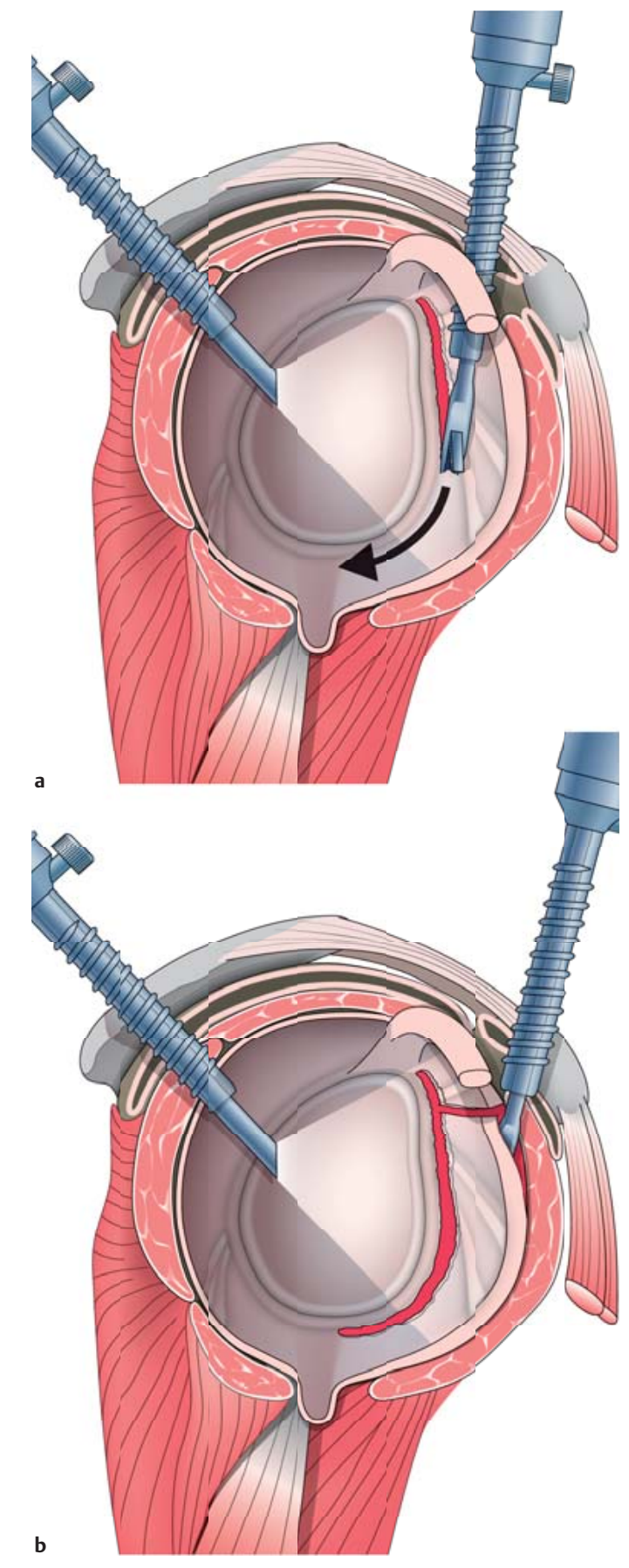

Diese Maßnahme lässt sich üblicherweise nach kaudal bis ca. 6.00 Uhr fortführen. Ventral/kaudal kann nach Entfernung der vorderen Kapsel breitflächig die dann freiliegende Subskapularismuskulatur gesehen werden.

Im Bereich des unteren Glenoids können die Voraussetzungen für die Arthrolyse verbessert werden, wenn der Assistent den Arm des Patienten gering außenrotiert und abduziert oder eine Tuchrolle unter die Axilla des Patienten geklemmt wird, um das Gelenk zu distrahieren.
MGHL und ventrale Kapsel, die normalerweise einem dünnen und zarten Bindegewebe entsprechen, können bei der Schultersteife bis auf $1 \mathrm{~cm}$ verdickt und beim Durchtrennen von derbem Charakter sein.

- Aufgrund der engen Lagebeziehung zum N. axillaris sollte sich die Inzision/Resektion der kaudalen Kapsel sehr eng am Glenoid bzw. am kaudalen Labrum orientieren.

Die Arthrolyse kann rein mechanisch über arthroskopische Fass- bzw. Resektionszangen oder Scheren (Abb. 8) oder elektrisch mit Hochfrequenzablation erfolgen (Abb. 9). Bei Verwendung von Strom können Kontraktionen des M. deltoideus die räumliche Nähe zum $\mathrm{N}$. axillaris anzeigen. Eine Inzision der posteroinferioren oder gar posterioren Kapsel ist über den anterioren $\mathrm{Zu}$ gang nicht möglich. Das Release des Rotatorenintervalls sollte als letzte der intraartikulären Maßnahmen erfolgen, da anschließend mit einer raschen Zunahme der Schwellung durch Extravasat der Spülflüssigkeit gerechnet werden muss. Eine Resektion des lateralen SGHL bzw. der medialen Bizepssehnenschlinge muss vermieden werden.

Dann (wie auch bereits zuvor zwischendurch) wird die Beweglichkeit überprüft. Die Kapsulotomie sollte keinesfalls übertrieben werden; bei Wiedererlangen eines normalen Bewegungsumfangs kann der intraartikuläre Anteil der Operation direkt beendet werden. Ist nach ventraler Kapsulotomie wieder eine gute Außenrotation vorhanden, kann eine vorsichtige geschlossene Mobilisierung des Schultergelenks in die Flexion erfolgen. Hierdurch kommt es typischerweise nach ventraler Kapsulotomie bis $6.00 \mathrm{Uhr}$ zu einem weiteren Einriss der posteroinferioren Kapsel von ca. 6.00-8.00 Uhr (Abb. 10), und anschließend ist auch die Flexion passiv wieder ohne Widerstand möglich.

Bei nur gering eingeschränkter Innenrotation wird auf weitere Maßnahmen verzichtet. Bei ausgeprägtem Innenrotationsdefizit muss für die dorsale Kapsulotomie dann (über einen Wechselstab) die Optik in das anteriore Portal eingeführt werden. Unter Sicht von ventral wird analog zur vorderen Kapsel nun die dorsale Kapsel parallel zum Labrum reseziert, bis auch die Innenrotation wieder frei möglich ist.

Schneller als bei anderen arthroskopischen Eingriffen am Schultergelenk kann es im Rahmen einer Arthrolyse zu einem Flüssigkeitsaustritt aus dem Gelenk mit zunehmendem Weichteilödem und dann durch die Schwellung schlechteren Operationsbedingungen kommen. Wichtig 

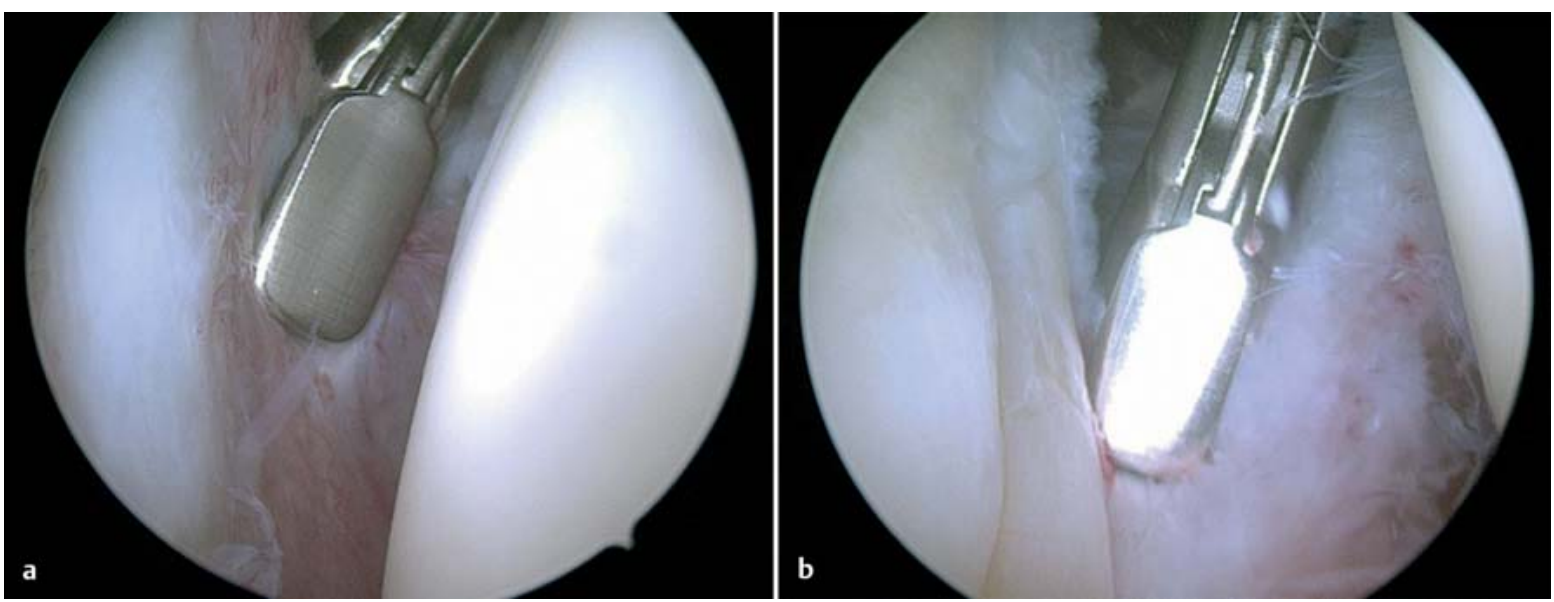

Abb. 8 - Arthroskopische Arthrolyse mit mechanischen arthroskopischen Fass- bzw. Resektionszangen. a Inzision bzw. Resektion des MGHL b Resektion der ventralen Gelenkkapsel.
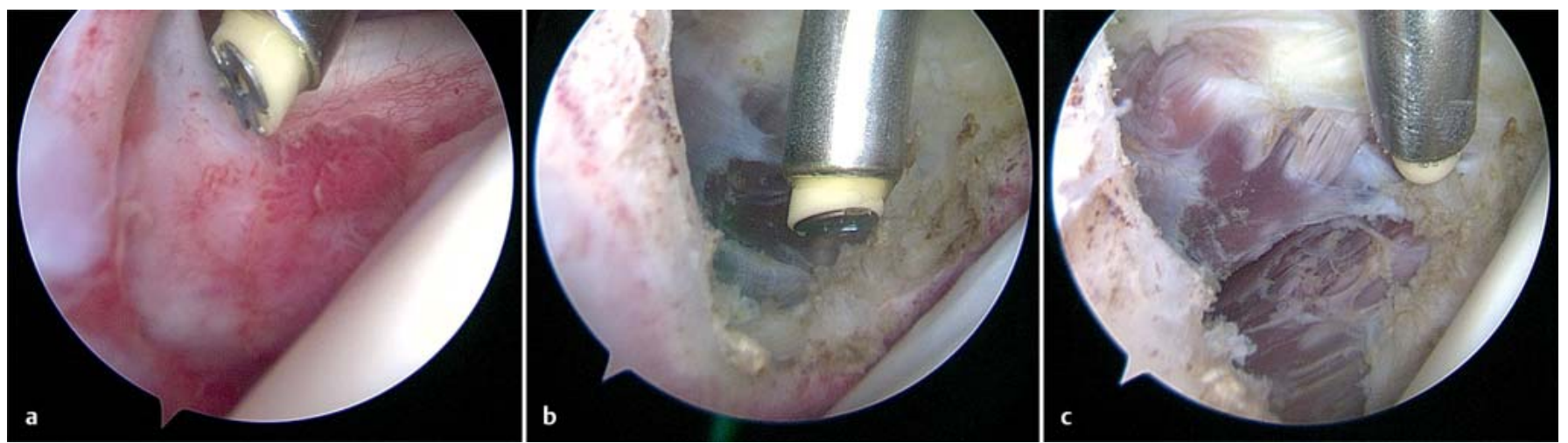

Abb. 9 - Arthroskopische Arthrolyse mittels Hochfrequenzablation. a Resektion des MGHL. b Resektion der ventralen Gelenkkapsel. c Zuletzt breitflächig freiliegende SSC-Sehne bzw. -Muskulatur.

ist daher eine gewisse Erfahrung, um die Operation zügig durchführen zu können. Es empfiehlt sich, für Zugänge das Gelenk stumpf zu perforieren und keine Inzisionen der Gelenkkapsel zu machen.

Der anteriore Zugang oberhalb der SSC-Sehne wird unter arthroskopischer Sicht nach Probepunktion mit einer Kanüle angelegt (Outside-in-Technik). Wichtig ist hierbei eine weit mediale Lokalisation des Zugangs direkt lateral des Processus coracoideus und in Verlängerung der Glenoidebene im Gegensatz zum eher lateralen Zugang zwischen SSC-Ansatz und medialer Schlinge für die ventrale Stabilisierung. Nur bei ausreichend medialem Zugang kann eine Kapsulotomie parallel zum Labrum ventral von kranial beginnend bis zur oder über die 6.00-Uhr-Position des Glenoids hinaus durchgeführt werden.

Nach Beenden der intraartikulären Arbeitsschritte wird abschließend über ein laterales Portal noch die endoskopische subakromiale Bursektomie durchgeführt. Entzündetes Gewebe wird entfernt, und Verwachsungen werden

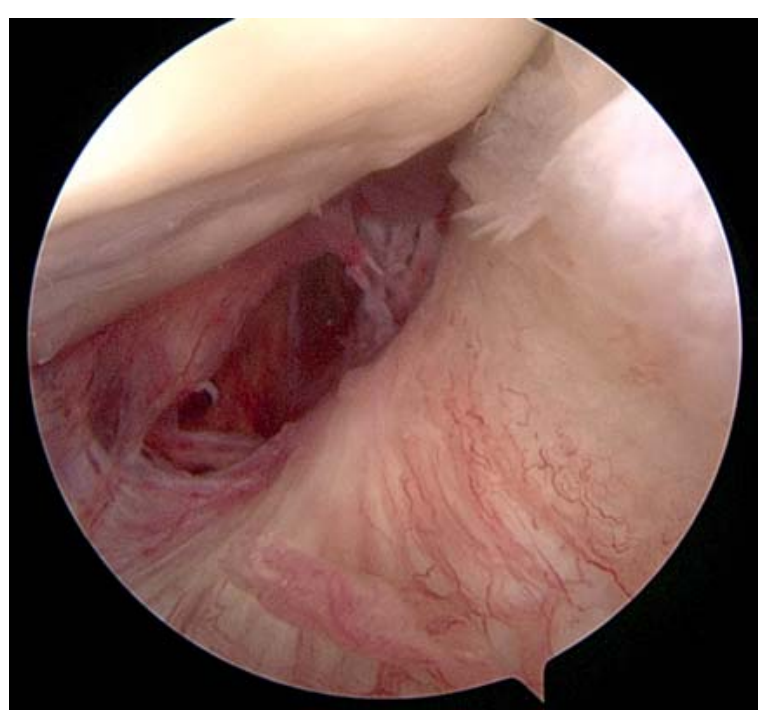

Abb. 10 - Typisches Bild einer Zerreißung der posterokaudalen Gelenkkapsel (von ca. 6.00-8.00 Uhr) nach Mobilisierung (Blick von dorsal, rechtes Schultergelenk, Humeruskopf und dorsales Labrum oben im Bild). 
Übersicht

\section{Komplikationen der arthroskopischen Arthrolyse}

- (Nach-)Blutung/Hämarthros/Revision

- postoperative Infektion

- Risiko von iatrogenen Knorpel- oder Sehnenschäden

- fehlender Erfolg/insuffizientes Release/keine (ausreichende) Verbesserung der Beweglichkeit

- N.-axillaris-Schädigung (mit Folgeoperationen bzw. ausgeprägter Funktionseinschränkung bei Deltaparese)

gelöst (insbesondere bei der sekundären Steife sind oft Adhäsionen vorhanden). Bei engem Gleitraum oder Spornbildung kann wie beim klassischen Subakromialsyndrom eine Akromioplastik zusätzlich erfolgen. Bei der primären Steife ist der Gleitraum oft unauffällig als Zeichen der kapsulären/intraartikulären Pathologie der Erkrankung. Studien konnten hier eine zwingende Notwendigkeit eines zusätzlichen subakromialen Eingriffs nicht immer bestätigen. Bei geringem Aufwand und fehlenden Komplikationen sowie besserer Beurteilbarkeit der Rotatorenmanschette und des gesamten Krankheitsbildes ist dieser Teil jedoch auch bei der frozen shoulder zu empfehlen, bei der sekundären Steife selbstverständlich.

Da die klinisch oft vorhandenen Schmerzen am ACGelenk meist reaktiv sind, wird auf eine zusätzliche AC-Resektion verzichtet, anhaltende AC-Beschwerden sind nicht zu erwarten.

Komplikationen. Die Komplikationen, die bei einer arthroskopischen Arthrolyse auftreten können und beim

\footnotetext{
Abb. 11 - Patientin nach erfolgter arthroskopischer Arthrolyse links am ersten postoperativen Tag mit selbstständiger passiver Übung zum Erhalt der verbesserten Flexion unter Einsatz der Gegenhand.
}

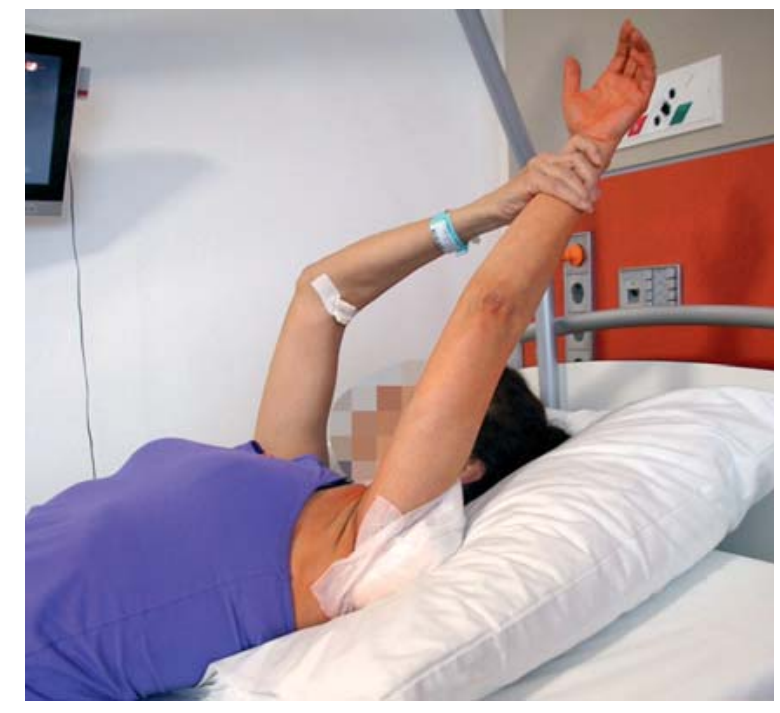

Aufklärungsgespräch besprochen (und dokumentiert) werden müssen, sind in der Übersicht aufgeführt.

\section{Nachbehandlung}

- Die postoperative Nachbehandlung ist der wichtigste Teil der operativen Therapie.

Oft lässt sich arthroskopisch sowohl bei der primären als auch bei der sekundären Schultersteife wieder eine gute bzw. normale passive Beweglichkeit des Schultergelenks erreichen. Dann ist entscheidend, ob es dem Patienten gelingt, diese Verbesserung auch über die Zeit nach der Operation hinaus zu erhalten.

\section{- Bewegungsübungen}

Deshalb sind der rasche Beginn und die intensive Ausführung von Bewegungsübungen direkt im Anschluss an die Operation von größter Wichtigkeit. Bereits am OP-Tag sollten die Patienten mit eigenständigen Übungen beginnen. Der Operateur kann einige Stunden nach der Operation im Gespräch mit dem Patienten von den intraoperativen Befunden und durchgeführten Maßnahmen berichten und ihm die verbesserte Beweglichkeit des Schultergelenks demonstrieren. Dies hat einen positiven und motivierenden Effekt. Eine Anleitung zur selbstständigen Durchführung von Übungen wird gegeben, kurze - z.B. stündliche - Intervalle werden empfohlen. Bevorzugt in die Flexion, aber auch in die Rotation bzw. in allen Ebenen ist eine Mobilisierung sinnvoll.

\section{- Schmerztherapie}

Hierzu ist auch eine begleitende ausreichende postoperative Schmerztherapie wesentlich. Wenn Patienten aus Schmerzgründen nicht intensiv bewegen können, ist der Erfolg des Eingriffs gefährdet. Es bietet sich zusätzlich zur oralen postoperativen Analgetikagabe die Schmerztherapie mit einem Plexuskatheter an, der nach Bedarf befahren werden kann. Bei hiermit oft vollständig schmerzfreier Schulter und paretischem Arm können die Übungen dann passiv unter Zuhilfenahme der Gegenhand ausgeführt werden (Abb. 11).

Aus diesem Grund ist es auch erforderlich, dass der Eingriff unter stationären Bedingungen erfolgt, da ambulant eine ausreichende Analgesie nicht gewährleistet werden kann. Nach 2-3 Tagen ist der Katheter dann meist nicht mehr notwendig und eine orale Medikation ausreichend. Dann kann die weitere Nachbehandlung ambulant erfolgen. 


\section{- Physiotherapie}

Weiterhin sind mehrfach täglich eigene Übungen und eine intensive - am besten tägliche - Physiotherapie zweckmäßig. Es kann schmerzabhängig der Übergang zu aktiv-assistiven und auch aktiven Übungen erfolgen. Passive Dehnungen durch den Therapeuten oder z. B. auch an der Wand oder mit einem Seil/Theraband bleiben aber entscheidend, wobei die Schmerzgrenze erreicht werden sollte („no pain, no gain“).

Ein Schulterstuhl mit CPM („continuous passive motion“) kann begleitend eingesetzt werden, die Dehnung im endgradigen Bereich ist hier jedoch oft nicht im gleichen Ausmaß möglich.

\section{Weiterer Verlauf}

Das Erreichen einer weitgehend schmerzfreien Schulterfunktion dauert üblicherweise ca. 6 Wochen, dann sind Arbeiten im Bereich unterhalb der Horizontalen und z.B. Bürotätigkeiten wieder ausführbar. Bei Berufen mit körperlicher Belastung kann die Dauer der Arbeitsunfähigkeit auf 2-3 Monate steigen. Physiotherapie ist teils auch darüber hinaus noch erforderlich.

Bis sich das Endergebnis einstellt, vergehen in der Regel ca. 3-6 Monate.

Interessenkonflikt: Die Autoren bestätigen, dass kein Interessenkonflikt vorliegt.

\section{Korrespondenzadresse}

Dr. Friedrich Dehlinger

Arcus Sportklinik Pforzheim

Rastatter Straße 17

75179 Pforzheim

Telefon: + $497231605-560$

Fax: $\quad+497231605-563089$

E-Mail: dehlinger@sportklinik.de

\section{Zum Weiterlesen und Vertiefen}

1 Berndt T, Elki S, Sedlinsch A et al. Arthroscopic release for shoulder stiffness. Oper Orthop Traumatol 2014; epub ahead of print

2 Codman EA. Tendinitis of the short rotators. In: Codman EA, ed. Ruptures of the Supraspinatus Tendon and other Lesions on or about the Subacromial Bursa. Boston, Mass: Thomas Todd; 1934

3 Denard PJ, Lädermann A, Burkhart SS. Prevention and management of stiffness after arthroscopic rotator cuff repair: systematic review and implications for rotator cuff healing. Arthroscopy 2011; 27: 842 -848

4 Favejee MM, Huisstede BM, Koes BW. Frozen shoulder: the effectiveness of conservative and surgical interventions-systematic review. Br J Sports Med 2011; 45: 49-56
5 Habermeyer P, Lichtenberg S, Magosch P. Schulterchirurgie, 4. Aufl. München: Urban und Fischer Verlag/Elsevier; 2010

6 Hand C, Clipsham K, Rees JL et al. Long-term outcome of frozen shoulder. J Shoulder Elbow Surg 2008; 17: 231-236

7 Hertel R. The frozen shoulder. Orthopäde 2000; 29: 845 - 851

8 Katthagen JC, Jensen G, Voigt C et al. Schultersteife. Unfallchirurg 2012; 115: 527-538

9 Levine WN, Kashyap CP, Bak SF et al. Nonoperative management of idiopathic adhesive capsulitis. J Shoulder Elbow Surg 2007; 16: $569-$ 573

10 Loew M, Heichel TO, Lehner B. Intraarticular lesions in primary frozen shoulder after manipulation under general anesthesia. J Shoulder Elbow Surg 2005; 14: 16-21

11 Lundberg BJ. The frozen shoulder: clinical and radiographical observations, the effect of manipulation under general anesthesia, structure and glycosaminoglycan content of the joint capsule, local bone metabolism. Acta Orthop Scand Suppl 1969; 119: 1-59

12 Mengiardi B, Pfirrmann CW, Gerber C et al. Frozen shoulder: MR arthrographic findings. Radiology 2004; 233: 486-492

13 Mehta SS, Singh HP, Pandey R. Comparative outcome of arthroscopic release for frozen shoulder in patients with and without diabetes. Bone Joint J 2014; 96: 1355-1358

14 Neviaser JS. Adhesive capsulitis of the shoulder. J Bone Joint Surg Am 1945; 27: 211-222

15 Page P, Labbe A. Adhesive capsulitis: use the evidence to integrate your interventions. N Am J Sports Phys Ther 2010; 5: 266-273

16 Raykha CN, Crawford JD, Burry AF et al. IGF2 expression and $\beta$-catenin levels are increased in Frozen Shoulder Syndrome. Clin Invest Med 2014; 37: E262-E267

17 Rockwood C, Matsen F. The Shoulder. 4th ed. Philadelphia: WB Saunders; 2009

18 Sung CM, Jung TS, Park HB. Are serum lipids involved in primary frozen shoulder? A case-control study. J Bone Joint Surg Am 2014; 96 : $1828-1833$

19 Zuckerman JD, Rokito A. Frozen shoulder: a consensus definition. J Shoulder Elbow Surg 2011; 20: 322-325

\section{Quellenangaben}

1 Cyriax J. Textbook of orthopaedic Medicine. Diagnosis of soft Tissue Lesions. Vol. 1. London: Balliere Tindall; 1982

2 Gartsman G. Shoulder Arthroscopy. 2nd ed. Philadelphia: WB Saunders; 2008

3 Habermeyer P, Dietz SO, Magosch P. Orale Cortisonstufentherapie zur Behandlung der Frozen shoulder. Ein neues stadiengerechtes konservatives Therapieschema. ATOS News 2003; 3: 12-15

4 Hawkins RJ, Kennedy JC. Impingement syndrome in athletes. Am J Sports Med 1980; 8: 151-158

5 Kessel L. Clinical Disorders of the Shoulder. New York: Churchill Livingstone; 1982

6 Matsen III FA, Lippitt SB, Sidles JA et al. Practical Evaluation of Management of the Shoulder. Philadelphia: WB Saunders; 1994: 19109

7 Neer C. Shoulder Reconstruction. Philadelphia: WB Saunders; 1990: $422-427$

8 Reeves B. The natural history of the frozen shoulder syndrome. Scand J Rheumatol 1975; 4: 193 -196 


\section{Schultergürtel und obere Extremität}

\section{CME-Fragen}

Welche Aussage zur

Pathophysiologie der

Schultersteife ist nicht richtig?

\section{CME•thieme.de}

\section{CME-Teilnahme}

- Viel Erfolg bei Ihrer CME-Teilnahme unter http://cme.thieme.de

- Bitte informieren Sie sich vorab online über die Gültigkeitsdauer.

- Sollten Sie Fragen zur Online-Teilnahme haben, unter http://cme.thieme.de/hilfe finden Sie eine ausführliche Anleitung.

A Ursache der Bewegungseinschränkung ist eine Fibrosierung der Gelenkkapsel

B Das intraartikuläre Volumen - z. B. gemessen mit einer Arthrografie - ist vergrößert.

C Die primäre Schultersteife wird im Englischen als „frozen shoulder“ bezeichnet.

D Die primäre Schultersteife kann folgenlos ausheilen.

E Die sekundäre Form der Schultersteife kann durch eine Kalkschulter ausgelöst werden.
A Die Erkrankung beginnt mit einer schmerzlosen Bewegungseinschränkung.

B Die Schmerzen nehmen von Phase 1 bis 3 stetig zu.

C In der 1. und 2. Phase sind orale Steroide nutzlos.

D Operationsindikationen werden unabhängig von den Phasen getroffen.

E Bei der sekundären Schultersteife sind die Phasen oft weniger eindeutig.
Die primäre Schultersteife nimmt oft einen phasenhaften Verlauf. Welche Aussage ist richtig?
Welche Situation aus der Schultersprechstunde ist falsch wiedergegeben?
A Bei der körperlichen Untersuchung der Schulter einer 52-jährigen Lehrerin fällt Ihnen ein gestörter skapulothorakaler Rhythmus auf. Dies ist ein Hinweis auf eine Schultersteife.

B Die Untersuchung eines 63-jährigen Waldarbeiters ergibt bei sonst seitengleichen Werten für die Flexion und die Innenrotation eine Außenrotation von $30^{\circ}$ links und $70^{\circ}$ rechts. Dies kann ein Zeichen einer alten SSC-Ruptur rechts sein.

C Bei einer 49-jährigen Büroangestellten, die seit 4 Wochen über spontan zunehmende Schulterschmerzen rechts klagt und deren Bewegungsumfang aufgrund der Schmerzen kaum untersucht werden kann, ist eine primäre Schultersteife ursächlich möglich.

D Ein 51-jähriger LKW-Fahrer stellt sich zur Kontrolle 4 Monate nach Rotatorenmanschettennaht vor. Schmerzen bestehen kaum, die passive Beweglichkeit der operierten Schulter wird mit Flexion $100^{\circ}$ (Gegenseite $170^{\circ}$ ) und Außenrotation $10^{\circ}$ (Gegenseite $50^{\circ}$ ) mit jeweils hartem Anschlag gemessen. Eine postoperative Schultersteife kann ausgeschlossen werden.

E Eine 72-jährige Patientin klagt über Schmerzen und eine zunehmend schlechte Beweglichkeit der rechten Schulter. Sie finden eine aktive Flexion von $90^{\circ}$ und eine passive Flexion von $170^{\circ}$. Es liegt hier keine Schultersteife, sondern der Verdacht auf einen Rotatorenmanschettendefekt vor. 
Welche Aussage zur bildgebenden Diagnostik bei der Schultersteife ist richtig?
4

A Eine wichtige Differenzialdiagnose, die radiologisch abgeklärt werden muss, ist die primäre Omarthrose.

B Die Röntgendiagnostik bei der primären Schultersteife zeigt einen verschmälerten Gelenkspalt.

C Sonografisch kann die Verdickung des MGHL gut dargestellt werden.

D Die MRT kann genau das Ausmaß der Entzündung und damit der Bewegungseinschränkung aufzeigen.

E Auf bildgebende Diagnostik kann vollständig verzichtet werden.
Wodurch ist die Therapie der Schultersteife gekennzeichnet?
A Im ersten Stadium der frozen shoulder sollte wegen der ausgeprägten Schmerzen rasch eine operative Therapie eingeleitet werden.

B In der 2. und 3 Phase kann Physiotherapie zur Besserung der Beweglichkeit eingesetzt werden.

C Bei der sekundären Schultersteife müssen die Bewegungseinschränkungen akzeptiert werden.

D Nur ca. $1 \%$ der Patienten wird einer operativen Therapie zugeführt.

E Orale Kortisontherapie ist bei der sekundären Schultersteife wirksamer als bei der primären.
Eine der folgenden Aussagen zur arthroskopischen Arthrolyse ist unzutreffend. Welche?
A Es kann sich eine Synovitis zeigen.

B Das MGHL kann narbig verdickt sein.

C Das vordere Labrum wird abgelöst.

D Der N. axillaris ist gefährdet.

E Eine zunehmende Schwellung kann die Operation erschweren.
Welche Aussage zur Therapie der Schultersteife ist falsch?
A Bei schmerzhaftem AC-Gelenk muss immer auch eine AC-Resektion durchgeführt werden.

B Eine Durchtrennung des MGHL führt zu einer verbesserten Außenrotation.

C Bei begleitendem Diabetes mellitus wird eine orale Kortisontherapie nicht empfohlen.

D Ein Plexuskatheter ist für die postoperative Schmerztherapie hilfreich.

E Physiotherapie ist in der 1. Phase der frozen shoulder oft mit einer Verschlechterung der Schmerzen verbunden.
Eine der Aussagen zur Epidemiologie der Schultersteife ist falsch. Welche?
A Die Inzidenz der frozen shoulder liegt bei ca. $2 \%$.

B Ein beidseitiges Auftreten der frozen shoulder wird in $20-30 \%$ beobachtet.

C Die Erkrankungshäufigkeit steigt mit zunehmendem Alter.

D Ein gehäuftes gleichzeitiges Auftreten eines Morbus Dupuytren ist bekannt.

E Diabetiker haben ein erhöhtes Risiko für die Entwicklung einer Schultersteife. 


\section{Schultergürtel und obere Extremität}

Zu den therapeutischen Möglichkeiten der Schultersteife gehört nicht...
A ... in der ersten Phase der frozen shoulder die Gabe von NSAID.

B ... der rasche Beginn und die intensive Ausführung von Bewegungsübungen direkt im Anschluss an eine OP.

C ... bei fehlender Besserung unter konservativer Therapie die arthroskopische Arthrolyse.

D ... eigenständiges Üben durch den Patienten (Dehnübungen) bei der sekundären Schultersteife.

E ... die dauerhafte Kortisontherapie.
Eine der folgenden Aussagen ist falsch. Welche?
A Die Krankheitsdauer bei der frozen shoulder kann 2 Jahre betragen.

B Lange postoperative oder posttraumatische Ruhigstellung des Schultergelenks kann die Entwicklung einer Steifigkeit begünstigen.

C Narben und Adhäsionen im subakromialen Gleitraum können ähnliche Symptome wie eine Kapsulitis verursachen.

D Beim Kapselmuster nach Cyriax ist zunächst die Innenrotation betroffen.

E Die Impingement- und Rotatorenmanschettentests sind bei der frozen shoulder oft positiv, obwohl kein typisches Impingement-Syndrom und kein Rotatorendefekt vorliegen. 